\title{
Single top production in PDF fits
}

\author{
Emanuele R. Nocera, ${ }^{a}$ Maria Ubiali $^{b}$ and Cameron Voisey ${ }^{c}$ \\ ${ }^{a}$ Nikhef, \\ Science Park 105, NL-1098 XG Amsterdam, The Netherlands \\ ${ }^{b}$ DAMTP, University of Cambridge, \\ Wilberforce Road, Cambridge, CB3 0WA, United Kingdom \\ ${ }^{c}$ Cavendish Laboratory (HEP), \\ JJ Thomson Avenue, Cambridge, CB3 OHE, United Kingdom \\ E-mail: e.nocera@nikhef.nl, M.Ubiali@damtp.cam.ac.uk, \\ voisey@hep.phy.cam.ac.uk
}

AbStRaCT: We study the impact of recent LHC $t$-channel single top-quark and topantiquark measurements at centre-of-mass energies of 7,8 and $13 \mathrm{TeV}$ on the parton distribution functions (PDFs) of the proton. We consider, namely, total cross sections, topantitop cross section ratios, and differential distributions. We present a critical appraisal of the data, studying in particular how their description is affected by the theoretical details that enter the computation of the corresponding observables: QCD and electroweak higher-order corrections, the flavour scheme, and the value of the bottom-quark threshold. We perform a series of fits to the data within the NNPDF3.1 framework, whereby next-to-next-to-leading order QCD corrections are applied to single top measurements in a systematic way. We find that there exists an optimal combination of data that maximises consistency with the rest of the dataset, and efficiency in constraining the up, down and, partially, gluon PDFs.

KeYwords: QCD Phenomenology

ARXIV EPRINT: 1912.09543 


\section{Contents}

1 Introduction 1

2 Experimental data $\quad 3$

2.1 Total top-quark and top-antiquark cross sections and their ratios 3

2.2 Differential cross sections 3

3 Data-theory comparison $\quad 5$

3.1 QCD corrections 6

$\begin{array}{lll}3.2 & \text { EW corrections } & 12\end{array}$

$\begin{array}{lll}3.3 & \text { Heavy flavour schemes } & 13\end{array}$

3.4 Quantitative assessment of the agreement between theory and data 17

4 Impact of single top data on PDFs $\quad 20$

4.1 Observable-PDF correlations 21

4.2 Fit settings 23

4.3 Fit results 24

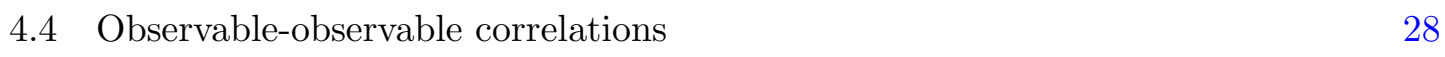

$\begin{array}{lll}4.5 & \text { Phenomenological impact } & 31\end{array}$

5 Conclusions $\quad 34$

A The ATLAS $8 \mathrm{TeV}$ differential distributions 36

\section{Introduction}

At hadron colliders, single top quarks and top antiquarks are produced in the Standard Model (SM) via the weak interaction involving, at leading order (LO), a Wtb vertex. Three channels contribute to this process: the exchange of a space-like $W$ boson, or $t$ channel $\left(q \bar{b} \rightarrow q^{\prime} \bar{t}\right.$ and $\bar{q} b \rightarrow \bar{q}^{\prime} t$ at LO); the exchange of a time-like $W$ boson, or $s$-channel $\left(q \bar{q}^{\prime} \rightarrow t \bar{b}, \bar{t} b\right.$ at LO); and the associated production of a top quark or antiquark with an on-shell $W$ boson $\left(g b \rightarrow t W^{-}\right.$and $g \bar{b} \rightarrow \bar{t} W^{+}$at LO). The $t$-channel leads to the largest cross section at the Large Hadron Collider (LHC).

Single top-quark and top-antiquark production is a signal to probe several aspects of the SM. For instance, it allows for the direct determination of the Cabibbo-KobayashiMaskawa (CKM) matrix element $\left|V_{t b}\right|$ without assuming unitarity $[1,2]$, and for the extraction of the top-quark mass $[3,4]$. It is also an important source of background events in searches for physics beyond the SM that could modify the structure of the $W t b$ vertex, or allow new gauge bosons or heavy quarks to interact via a flavour-changing neutral current [5-9]. Furthermore, single top-quark and top-antiquark production has also been 
suggested as a probe of parton distribution functions (PDFs), in particular to constrain the ratio of up and down-quark distributions at large momentum fractions $x$. The impact of measurements of inclusive total cross sections from the Tevatron and the LHC were specifically appraised in refs. [10, 11].

In this work, we study the impact of the $t$-channel single top-quark and top-antiquark production data on the PDFs of the proton in a systematic way. On the one hand, we will scrutinise the available experimental measurements, in particular from the LHC. We will focus on total top-quark and top-antiquark cross sections (or their ratio) from ATLAS [12-14] and CMS [15-17] at centre-of-mass energies of 7, 8 and $13 \mathrm{TeV}$; and on differential cross sections, both absolute and normalised to the total cross section, from ATLAS at $7 \mathrm{TeV}$ [12] and $8 \mathrm{TeV}$ [13]. On the other hand, we will investigate how the description of the data is affected by the theoretical details that enter the computation of single top observables. Specifically, we will pay attention to the inclusion of higher-order quantum chromodynamics (QCD) corrections [18-23], recently computed up to next-to-next-to-leading order (NNLO) accuracy [24-26]; to the effect of next-to-leading order (NLO) electroweak (EW) corrections [27-29]; and to the choice of the heavy flavour scheme. In this last respect we will restrict our analysis to NLO QCD, and compare the four-flavour scheme (4FS) [30], in which the bottom quark is treated as a massive final state and does not contribute to the proton wave-function, with the five-flavour scheme (5FS) [18], in which the bottom quark is treated as a light quark, perturbatively generated within the proton above a certain matching scale (often taken to be of the order of the bottom-quark pole mass). The phenomenological dependence on the exact value of the bottom-quark threshold, in particular on a higher bottom-quark matching scale as suggested in ref. [31], will be investigated as well.

The inclusion of single top-quark and top-antiquark data in a PDF fit will be realised in the framework of the NNPDF3.1 global analysis [32]. We will investigate the internal consistency of the new data (and the consistency of the new data with the rest of the NNPDF3.1 dataset); which theoretical details lead to its optimal description; whether it is advantageous to use absolute instead of normalised distributions; and which combination of LHC data maximises efficiency in constraining the PDFs. Such a combination will be suitable for inclusion in the next generation of global PDF fits.

The outline of the paper is as follows. In section 2 we present the experimental measurements of single top-quark and top-antiquark production that we will be studying. In section 3 we examine how this data is described upon various assumptions in the underlying theory. Specifically, we provide a quantitative assessment of the NNLO QCD corrections, of the NLO QCD $\times \mathrm{EW}$ corrections (in both cases computed within the $5 \mathrm{FS}$ ), and of NLO QCD corrections computed either in the $4 \mathrm{FS}$ or in the 5FS, possibly with a higher bottomquark matching scale. In section 4 we assess the impact of the data in a PDF fit: we study the correlations between the data and the various PDF flavours; we discuss the details of our fits; we formulate a recommendation on the data that, if included in a fit, maximises consistency with the rest of the dataset and efficiency in constraining the PDFs; we analyse the correlations between single top data and data that is already included in NNPDF fits as standard; and we assess the phenomenological implications of our optimal fit. Finally, 
we draw our conclusions in section 5. An additional investigation of the differential distributions from ATLAS at $8 \mathrm{TeV}$ is presented in appendix A. These data are not included in the fits because complete information on the experimental correlations within the data is not currently available.

\section{Experimental data}

In this section we present the $t$-channel single top-quark and top-antiquark production data from ATLAS and CMS that we study in this work. We first discuss measurements of top-quark and top-antiquark total cross sections and of their ratios, and we then discuss the absolute and normalised differential cross sections available as a function of various kinematic variables.

\subsection{Total top-quark and top-antiquark cross sections and their ratios}

The total cross section for $t$-channel single top production was first measured in protonantiproton collisions at a centre-of-mass energy of $1.96 \mathrm{TeV}$ by the D0 [33] and CDF [34] experiments at the Tevatron. These pioneering measurements established electroweak topquark production in hadronic collisions, gave insight into the properties of the $W t b$ vertex, and allowed for a first extraction of the CKM matrix element $\left|V_{t b}\right|$. However, because of the rather large statistical uncertainty in each case - a consequence of the low luminosities achieved - these measurements are not suitable for inclusion in modern PDF sets.

In this work, we therefore exclusively use the more precise measurements from the LHC. Such measurements were first made at a centre-of-mass energy of $7 \mathrm{TeV}$ by the ATLAS [35] and CMS [36] experiments, and have been subsequently complemented at a higher luminosity $[12,15]$, and at centre-of-mass energies of $8 \mathrm{TeV}[13,16]$ and $13 \mathrm{TeV}[14,17]$. In such analyses the top quark and top antiquark are reconstructed from a selection of their leptonic decay channels, $t \rightarrow e \nu b, t \rightarrow \mu \nu b$, and $t \rightarrow \tau \nu b$, with leptonic $\tau$ decays.

While total cross sections, $\sigma_{t+\bar{t}}$, are provided for all of the above measurements, individual cross sections for single top-quark and top-antiquark production, $\sigma_{t}$ and $\sigma_{\bar{t}}$, and their ratio, $\sigma_{t} / \sigma_{\bar{t}}$, are available only for the ATLAS measurements [12-14], and for the CMS measurements at $8 \mathrm{TeV}[16]$ and $13 \mathrm{TeV}$ [17]. In these cases, we include the ratio $\sigma_{t} / \sigma_{\bar{t}}$ because its experimental and theoretical analysis largely benefits from uncertainty cancellations between the numerator and the denominator. For the CMS measurement at $7 \mathrm{TeV}$ [15], we include the total cross section $\sigma_{t+\bar{t}}$; we discard earlier measurements from both ATLAS and CMS at $7 \mathrm{TeV}[35,36]$, which are based on reduced luminosities, and are therefore affected by rather large statistical uncertainties. The data points for the total top-quark and top-antiquark cross sections, and their ratio, that are included in this work are summarised in table 1 , where we list their centre-of-mass energy, $\sqrt{s}$, their integrated luminosity, $\mathcal{L}$, the corresponding observable, $\mathcal{O}$, their measured value, and their reference.

\subsection{Differential cross sections}

We also study differential cross section measurements for $t$-channel single top-quark and top-antiquark production from ATLAS at a centre-of mass energy of $7 \mathrm{TeV}$ [12]. These 


\begin{tabular}{|lccccc|}
\hline Experiment & $\sqrt{s}[\mathrm{TeV}]$ & $\mathcal{L}\left[\mathrm{fb}^{-1}\right]$ & $\mathcal{O}$ & Measurement & Ref. \\
\hline ATLAS & 7 & 4.59 & $\sigma_{t} / \sigma_{\bar{t}}$ & $2.04 \pm 0.13$ (stat.) \pm 0.12 (syst.) & {$[12]$} \\
& 8 & 20.2 & $\sigma_{t} / \sigma_{\bar{t}}$ & $1.72 \pm 0.05$ (stat.) \pm 0.07 (syst.) & {$[13]$} \\
& 13 & 3.2 & $\sigma_{t} / \sigma_{\bar{t}}$ & $1.72 \pm 0.09$ (stat.) \pm 0.18 (syst.) & {$[14]$} \\
\hline CMS & 7 & 2.73 & $\sigma_{t+\bar{t}}$ & $67.2 \pm 6.1 \mathrm{pb}$ & {$[15]$} \\
& 8 & 19.7 & $\sigma_{t} / \sigma_{\bar{t}}$ & $1.95 \pm 0.10$ (stat.) \pm 0.19 (syst.) & {$[16]$} \\
& 13 & 2.2 & $\sigma_{t} / \sigma_{\bar{t}}$ & $1.81 \pm 0.18$ (stat.) \pm 0.15 (syst.) & {$[17]$} \\
\hline
\end{tabular}

Table 1. Measurements of total $t$-channel single top-quark and top-antiquark cross sections and their ratio analysed in this work. For each of them we indicate the experiment, the centre-ofmass energy, $\sqrt{s}$, the integrated luminosity, $\mathcal{L}$, the measured observable, $\mathcal{O}$, its value (stat. and syst. denote statistical and systematic uncertainties), and its reference.

measurements are provided as a function of the transverse momentum, $p_{T}(t)\left(p_{T}(\bar{t})\right)$, and the absolute value of the rapidity, $|y(t)|(|y(\bar{t})|)$, of the top quark (top antiquark), and are provided in two forms: one in which they are normalised to the corresponding total cross section evaluated by integrating over all kinematic bins, and one in which they are not. In the former case, we always explicitly remove the last bin of the distribution, because its value is fixed by the normalisation condition. In principle, the inclusion of the distributions in either form should have the same statistical impact in a fit of PDFs. In this respect, to avoid loss of information on the overall normalisation, we always accompany normalised distributions with the total cross sections corresponding to the same experimental analysis. In contrast, the simultaneous inclusion of total cross sections and absolute differential distributions would result in double counting, because these are both determined from the same data sample.

In practice, however, the inclusion of absolute or normalised distributions in a fit might make a difference, because some approximations have to be made in the analysis of the data. First, the experimental correlation between the differential distributions and the total cross section - which should be taken into account - is not available. We therefore assume that, because the total cross section amounts to a single data point, and its exclusion from the fit would make no difference, the neglect of such correlation will a fortiori make little difference. Second, the theoretical description of normalised distributions benefits from a partial cancellation of theoretical uncertainties that occurs in the ratio. Similarly to what happens for experimental uncertainties, such a cancellation would be compensated by the theoretical uncertainty on the total cross section and the associated correlations. While theoretical uncertainties could be included in a fit [37, 38], they are not in this study.

We account for all of the available information on systematic and statistical correlations. Systematic uncertainties are correlated, for each separate source, bin-by-bin within each distribution. Likewise, we also include bin-by-bin correlations of statistical uncertainties within each distribution. Systematic and statistical correlations across different distributions, and between normalised distributions and the total cross sections, are not determined experimentally, and are therefore not considered here. The only exception is 


\begin{tabular}{|lcc|}
\hline Distribution & $N_{\text {dat }}$ & Kinematic range \\
\hline$d \sigma / d p_{T}(t)$ & 5 & $0<p_{T}(t)<500 \mathrm{GeV}$ \\
$d \sigma / d p_{T}(\bar{t})$ & 5 & $0<p_{T}(\bar{t})<500 \mathrm{GeV}$ \\
$d \sigma / d|y(t)|$ & 4 & $0<|y(t)|<3$ \\
$d \sigma / d|y(\bar{t})|$ & 4 & $0<|y(\bar{t})|<3$ \\
$(1 / \sigma) d \sigma / d p_{T}(t)$ & 4 & $0<p_{T}(t)<500 \mathrm{GeV}$ \\
$(1 / \sigma) d \sigma / d p_{T}(\bar{t})$ & 4 & $0<p_{T}(\bar{t})<500 \mathrm{GeV}$ \\
$(1 / \sigma) d \sigma / d|y(t)|$ & 3 & $0<|y(t)|<3$ \\
$(1 / \sigma) d \sigma / d|y(\bar{t})|$ & 3 & $0<|y(\bar{t})|<3$ \\
\hline
\end{tabular}

Table 2. The ATLAS measurements [12] of $t$-channel single top-quark and top-antiquark differential cross sections at a centre-of-mass energy of $7 \mathrm{TeV}$ studied here. Distributions are a function of the top-quark (top-antiquark) transverse momentum, $p_{T}(t)\left(p_{T}(\bar{t})\right)$, and absolute rapidity, $|y(t)|$ $(|y(\bar{t})|)$. The bin edges for the transverse momentum distributions are $0,45,75,110,150$ and $500 \mathrm{GeV}$, while for the rapidity distributions they are $0,0.2,0.6,1.1$ and 3.0. For each distribution, we indicate the number of bins, $N_{\text {dat }}$, and their overall kinematic range.

the luminosity uncertainty (for absolute distributions and the total cross section), which is $100 \%$ correlated across all of the ATLAS measurements at the same centre-of-mass energy. Because of this partial knowledge of correlations, and to avoid double counting, only one of top-quark distributions and one of top-antiquark distributions can be included in a fit at a time. In table 2 we summarise the features of each kinematic distribution, indicating whether it is an absolute or a normalised distribution, the number of data points, $N_{\text {dat }}$, and its kinematic coverage.

Finally, we shall note that other measurements of differential distributions for $t$-channel single top-quark and top-antiquark production have become available recently. Namely, the ATLAS measurements at a centre-of-mass energy of $8 \mathrm{TeV}$ [13], and the CMS measurements at a centre-of-mass energy of $8 \mathrm{TeV}$ [39] and $13 \mathrm{TeV}$ [40]. However, because no experimental information is provided on the individual sources of systematic uncertainty and their correlations for these measurements, they are not currently suitable for use in precision PDF determinations. Therefore, they are not included in the fits presented in section 4 . Nevertheless, we illustrate the $8 \mathrm{TeV}$ ATLAS data of ref. [13] in appendix A.

\section{Data-theory comparison}

In this section we scrutinise the description of the datasets discussed in section 2 upon various assumptions in the underlying theory. Specifically, we investigate the impact of QCD corrections, EW corrections, and the flavour scheme adopted, especially in relationship with the choice of the factorisation scale and, in the case of the 5FS, the bottom-quark threshold. Our study aims at identifying the optimal theoretical settings to include the data in a PDF fit. 


\subsection{QCD corrections}

Computations of NLO QCD corrections to $t$-channel single top-quark and top-antiquark production total cross sections were performed for the first time more than twenty years ago [18], and have been extended to fully differential cross sections [21], including decays [22, 23, 41, 42], since then. Monte Carlo simulations that are accurate to NLO have been implemented consistently within several generators, namely MADGRAPH5_AMC@NLO [43], Powheg [44], and Sherpa [45]. They all allow for the construction of fast interpolation grids, whereby partonic matrix elements are precomputed in such a way that the numerical convolution with generic input PDFs can be efficiently approximated by means of interpolation techniques. Such grids are a fundamental ingredient in any PDF fit, where the evaluation of the hadronic cross sections needs to be performed a large number of times.

We generate fast interpolation grids, accurate to NLO in QCD, for all of the $t$-channel single top-quark and top-antiquark data described in section 2. To this purpose, we use MADGRAPH5_AMC@NLO (v2.6.6 [46]) interfaced to APPLGRID [47] with AMCFAST [48]; the APPLGRID output is processed with APFELGRID [49] to construct fast interpolation grids in a format suitable for an NNPDF fit. The computation is performed in the 5FS with fixed factorisation and renormalisation scales $\mu_{f}=\mu_{r}=m_{t}$, where $m_{t}$ is the mass of the top quark, which we set equal to $172.5 \mathrm{GeV}$. The values of the bottom threshold, the $W$-boson mass, the $Z$-boson mass, and the strong coupling are chosen to be $m_{b}=4.92 \mathrm{GeV}$, $M_{W}=80.398 \mathrm{GeV}, M_{Z}=91.1876 \mathrm{GeV}$, and $\alpha_{s}\left(M_{Z}\right)=0.118$, respectively [32]. We require that the number of Monte Carlo events generated in the computation is sufficiently high to ensure that the residual relative statistical fluctuations are smaller than one permille; that is, that they are negligible with respect to the experimental precision of the data and the theoretical accuracy of the corresponding predictions. Our numerical results are benchmarked against the corresponding calculations obtained independently in ref. [26]. An excellent agreement, within the Monte Carlo statistical uncertainty, is found.

Very recently, NNLO QCD corrections to both total and differential cross sections have also been computed [24-26]. When the top decay is calculated, it is done in the narrow-width approximation, under which the QCD corrections to the top-(anti)quark production and the decay are factorisable. The full QCD corrections are approximated by the vertex corrections (a procedure known as the structure function approach in the inclusive case [50]). These calculations, however, are not available in a format suitable for their direct inclusion in a global fit of PDFs. In order to make use of them, we therefore resort to the $C$-factor approximation [51]: our NLO results computed with NNLO PDFs are multiplied by a bin-by-bin factor

$$
C=\frac{\hat{\sigma}_{\mathrm{NNLO}} \otimes \mathcal{L}_{\mathrm{NNLO}}}{\hat{\sigma}_{\mathrm{NLO}} \otimes \mathcal{L}_{\mathrm{NNLO}}}
$$

where $\hat{\sigma}_{\mathrm{NNLO}}\left(\hat{\sigma}_{\mathrm{NLO}}\right)$ is the partonic cross section computed with NNLO (NLO) matrix elements, and $\mathcal{L}_{\mathrm{NNLO}}$ is the corresponding parton luminosity evaluated with a reference set of NNLO PDFs. The numerator and the denominator in eq. (3.1) are computed with the code of refs. [25, 26] and the baseline NNPDF3.1 PDF set [32]. 


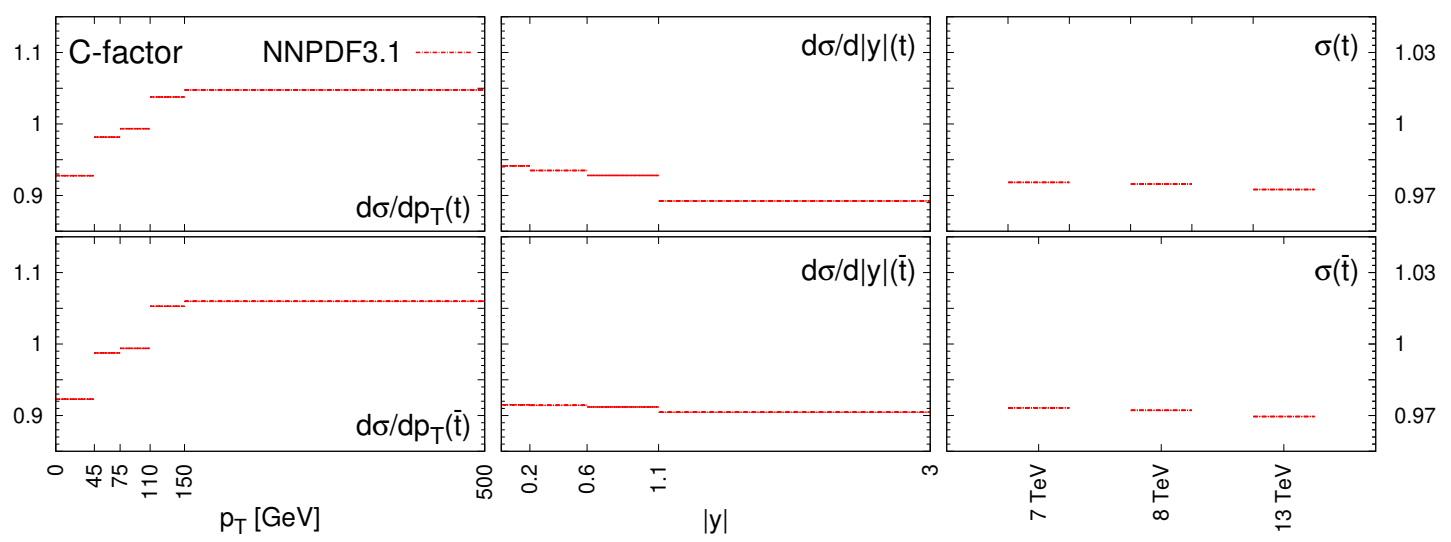

Figure 1. The $C$-factors, eq. (3.1), computed with the NNPDF31_nnlo_as_0118 PDF set, for the distributions differential in the transverse momentum (left), in the absolute rapidity (centre), and for the total cross sections (right) of top-quark (top) and top-antiquark (bottom). Results are displayed at $7 \mathrm{TeV}$ for differential distributions, and at 7,8 , and $13 \mathrm{TeV}$ for total cross sections. Note that the scale on the vertical axis varies: it ranges from 0.9 to 1.1 for the transverse momentum distributions, while it ranges from 0.97 to 1.03 for the rapidity distributions and for the total cross sections.

In figure 1 we display the $C$-factors computed according to eq. (3.1) relative to the datasets discussed in section 2 (see also tables 1-2): specifically, for the differential distributions in the transverse momentum and absolute rapidity of the top quark and of the top antiquark at $7 \mathrm{TeV}$, and for the total cross sections for the top quark and the top antiquark at 7,8 , and $13 \mathrm{TeV}$. We observe that NNLO corrections are comparable for top-quark and top-antiquark production. They display some structure for the transverse momentum distributions, where they lead to an enhancement of the cross section (up to about 5\%) at large values of the transverse momentum. Conversely, NNLO corrections display little sensitivity to the kinematics for the absolute rapidity distributions and for the total cross section; in these cases they lead to a suppression of the cross section (of about $3 \%$ ) across all of the measured ranges in rapidity and centre-of-mass energy.

We now compare theoretical predictions, computed as explained above at NLO and NNLO in pure QCD, to the experimental data listed in tables 1-2: in figure 2 we display the results for the total cross sections, in each case normalised to the central value of the data point; in figures 3-4 we display the results for the differential cross sections, both absolute (left) and normalised (right), for top-quark and top-antiquark production, respectively. For data-theory comparison purposes, we do not remove the last bin of the normalised distributions, opposite to what we do in a fit (see section 2.2), the reason being consistency with ref. [12]. For the differential distributions, the data and the theoretical predictions are normalised to the central value of the data in the central inset of each plot. The error bars on the data correspond to the square root of the diagonal elements of the experimental covariance matrix (see eq. (3.3)). The error bars (or bands) on theoretical predictions correspond to the $68 \%$ confidence level (CL) PDF uncertainty. The NNPDF31_nlo_as_0118 and NNPDF31_nnlo_as_0118 PDF sets are used for NLO and NNLO predictions, respectively. 


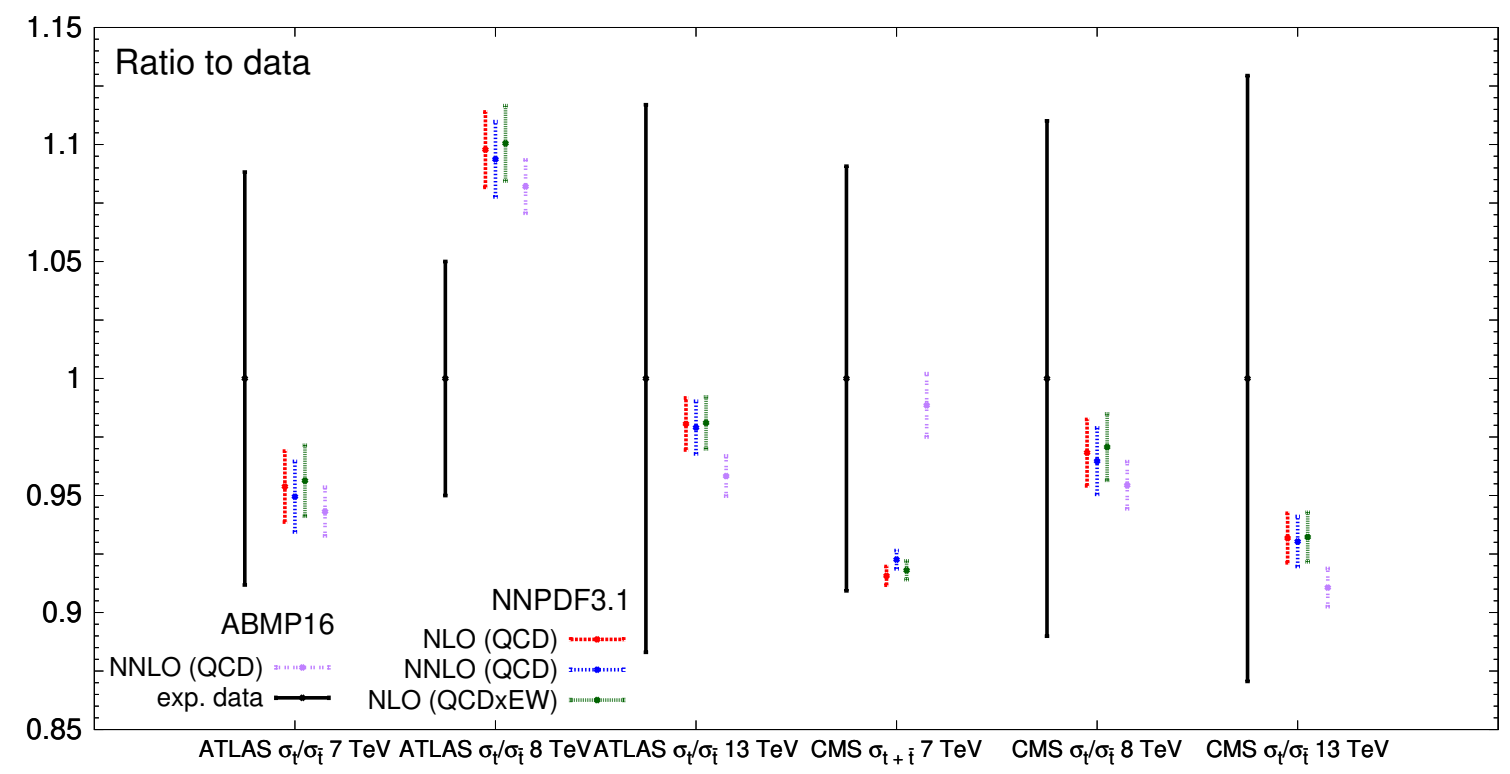

Figure 2. The total $t$-channel single top-quark and top-antiquark cross sections and their ratios from ATLAS and CMS analysed in this work (see table 1). The measurements are compared to theoretical predictions computed at NLO and NNLO in pure QCD, and at NLO in mixed QCD and EW theories. The corresponding sets of PDFs from the NNPDF3.1 determination are used: NNPDF31_nlo_as_0118, NNPDF31_nnlo_as_0118, and NNPDF31_nlo_as_0118_luxqed, respectively. For NNLO QCD theory, an alternative PDF set that includes some of the data in table 1, ABMP16als118_5_nnlo, is also used for comparison (see text for details). Both the data and the theoretical predictions are normalised to the central value of the data. Error bars on the data correspond to the square root of the sum in quadrature of statistical and systematic uncertainties; error bars on the theoretical predictions correspond to the $68 \%$ CL PDF uncertainty.

We note from figures 2-4 that NNLO QCD corrections are in general small, consistently with expectations based on $C$-factors (see figure 1 ). For the ratios of single top-quark to top-antiquark total cross sections their effect cancels out almost completely; for their sum (in the case of the CMS data point at $7 \mathrm{TeV}$ ), they increase the theoretical expectations by about $2 \%$, an amount which is smaller than both the data and the PDF uncertainties. For the distributions differential in the transverse momentum of the top quark (top antiquark), they can be as large as $10 \%$, especially at large values of $p_{T}(t)\left(p_{T}(\bar{t})\right)$, while for the distributions differential in the rapidity of the top quark (top antiquark), they never exceed 1-2\%. In both cases the NNLO QCD corrections display a similar size for the absolute and the normalised distributions. They result in a shift of the theoretical predictions always smaller than the data uncertainty, which remains rather larger than the PDF uncertainty. For this reason, we anticipate a moderate impact of single top-quark and top-antiquark data on PDFs when they are included in a fit (see section 4).

That being said, a qualitative inspection of figures $2-4$ reveals that theoretical predictions based on the NNPDF3.1 PDF sets are overall in fair agreement with the data. Significant discrepancies are observed only in two cases, consistently with what was qualitatively reported in ref. [12] (see in particular figures 15-16 therein). First, the ATLAS 

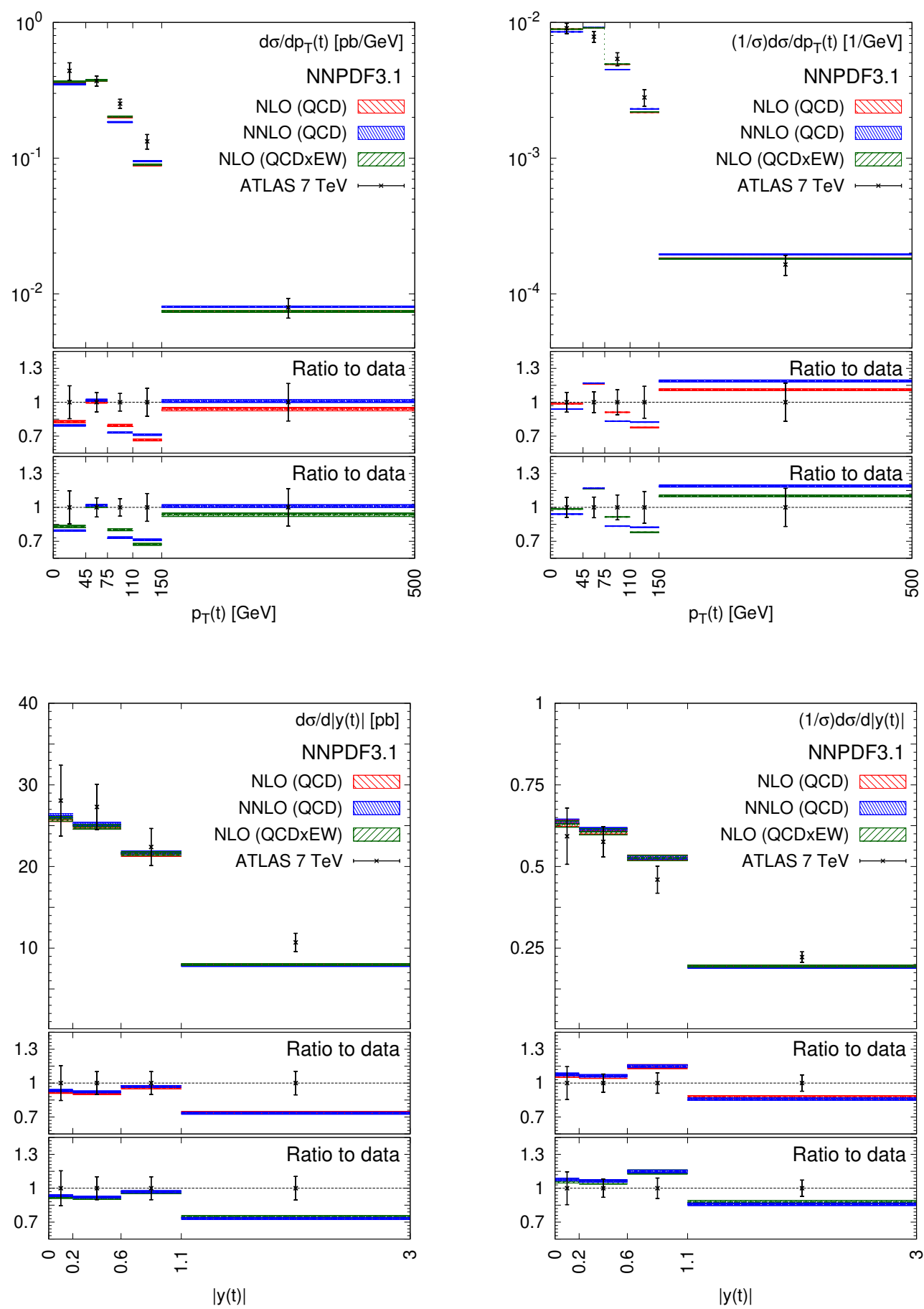

Figure 3. The $t$-channel single top-quark absolute (left) and normalised (right) cross sections from ATLAS analysed in this work (see table 2). The measurements are compared to theoretical predictions computed as in figure 2. The two lower panels display the data and the theoretical predictions normalised to the central value of the data. 

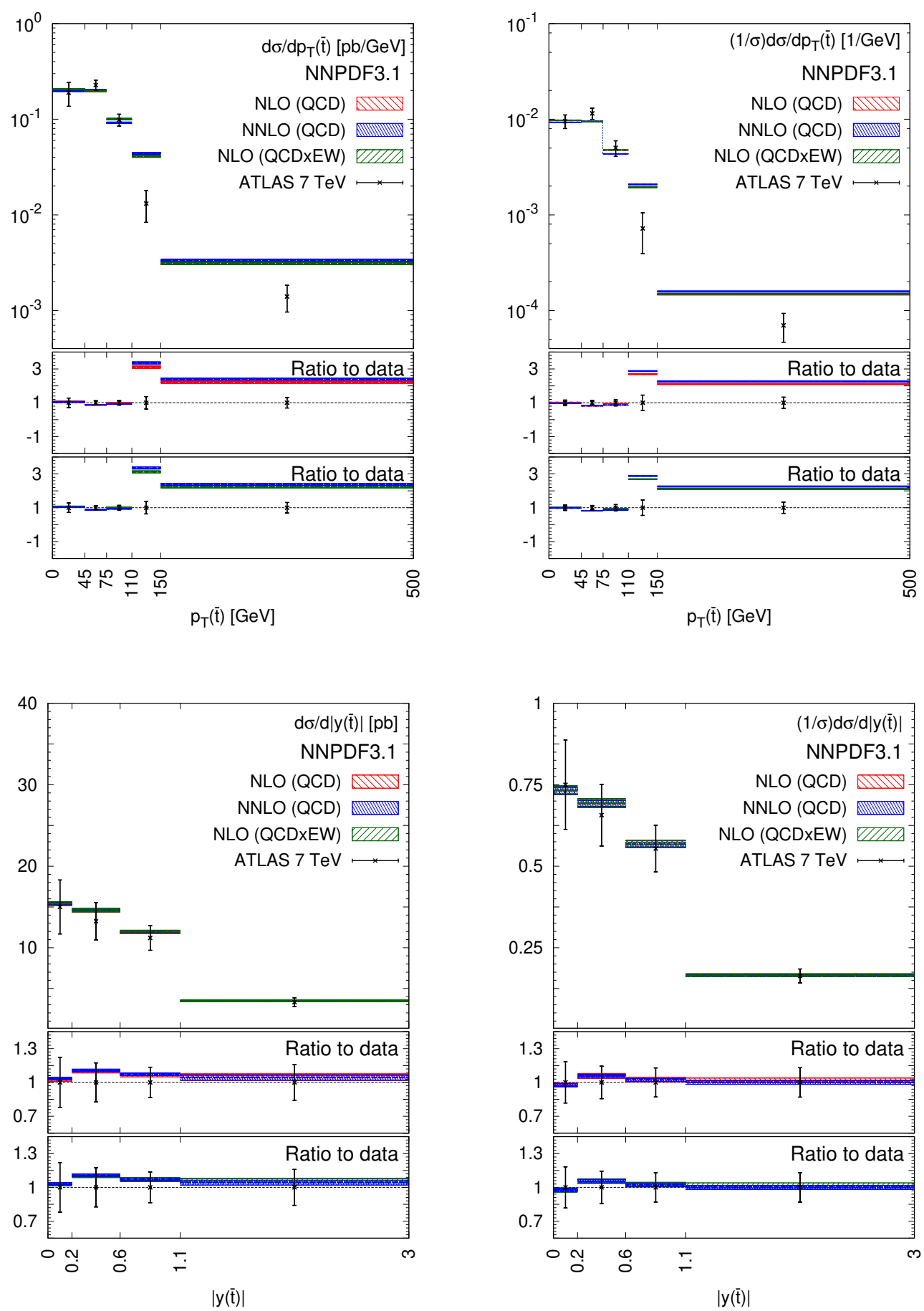

Figure 4. Same as figure 3, but for top-antiquark production. 
single top-quark to top-antiquark production cross section ratio at $8 \mathrm{TeV}$ differs from the theoretical expectation by about $10 \%$, corresponding to a $2 \sigma$ interval in units of the experimental uncertainty. Such a discrepancy is not observed for the analogous measurement from CMS: after all, it is apparent from table 1 that the ATLAS and CMS measurements at $8 \mathrm{TeV}$ have barely touching error bands, i.e. a $\sqrt{2} \sigma$ discrepancy. Second, the single top-antiquark cross section differential in the transverse momentum of the top-antiquark differs from the theoretical expectation by up to $300 \%$ for the two bins at the largest values of $p_{T}(\bar{t})$. This discrepancy is independent from the normalisation of the distribution, as it is present for both the absolute and the normalised cross sections. Furthermore, it is not alleviated by the inclusion of NNLO QCD corrections in the computation of the theoretical expectations.

It might be interesting to investigate whether these discrepancies persist if PDF sets other than NNPDF3.1 are used. We therefore look at the data-theory comparison obtained alternatively with the MMHT [52], CT18 [53], or the PDF4LHC15 combination [54]. None of these PDF sets include any single top data. Such comparisons appear to be almost identical to NNPDF3.1, therefore we do not report them explicitly in figures 2-4. For completeness, we also look at data-theory comparisons obtained with the ABMP16 PDF set [11]: this is the only PDF determination that includes some single top data, namely the total cross section for single top-quark production from the combined D0 and CDF measurements at $\sqrt{s}=1.96 \mathrm{TeV}[55]$ and from ATLAS [12, 14, 56] and CMS [15-17] measurements at $\sqrt{s}=7,8$ and $13 \mathrm{TeV}$. Specifically, we restrict ourselves to NNLO, and use the PDF set determined with five active flavours and a fixed value of $\alpha_{s}=0.118$, ABMP16als118_5_nnlo. We report such comparisons in figure 2 for the total cross section, and in figure 5 for the differential cross sections. In all cases, the data and the theoretical predictions are normalised to the central value of the data.

We see that the ABMP16 and the NNDPF3.1 predictions differ by an amount which depends on the specific distribution considered. For the total cross sections, the largest differences are observed for the single top-quark to single top-antiquark ratio at $\sqrt{s}=$ $13 \mathrm{TeV}$ (about $\sqrt{2} \sigma$ in units of the PDF uncertainty), and for the total top-quark and topantiquark cross section from CMS at $\sqrt{s}=7 \mathrm{TeV}$ (about $3 \sigma$ ). Such differences are always smaller than the data uncertainty. For the differential cross sections, similar differences are observed for all distributions, with ABMP16 predictions generally being closer to the

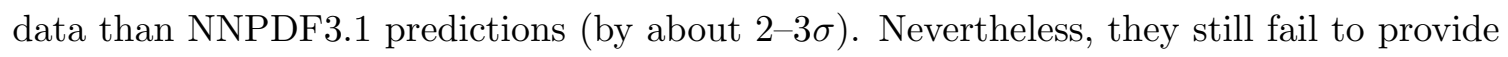
a good description of the ATLAS single top-quark to top-antiquark ratio measurement at $\sqrt{s}=8 \mathrm{TeV}$, and of the last two bins of the single top-antiquark absolute and normalised distributions differential in the transverse momentum of the top antiquark. We therefore conclude that the same discrepancies persist across any available PDF set.

The impact on these discrepancies of other theoretical details, such as the inclusion of EW corrections, the dependence on the flavour scheme, and the variation of the factorisation and the renormalisation scales will be studied in sections 3.2-3.3. A quantitative assessment of the agreement between data and theory will be provided in section 3.4. 

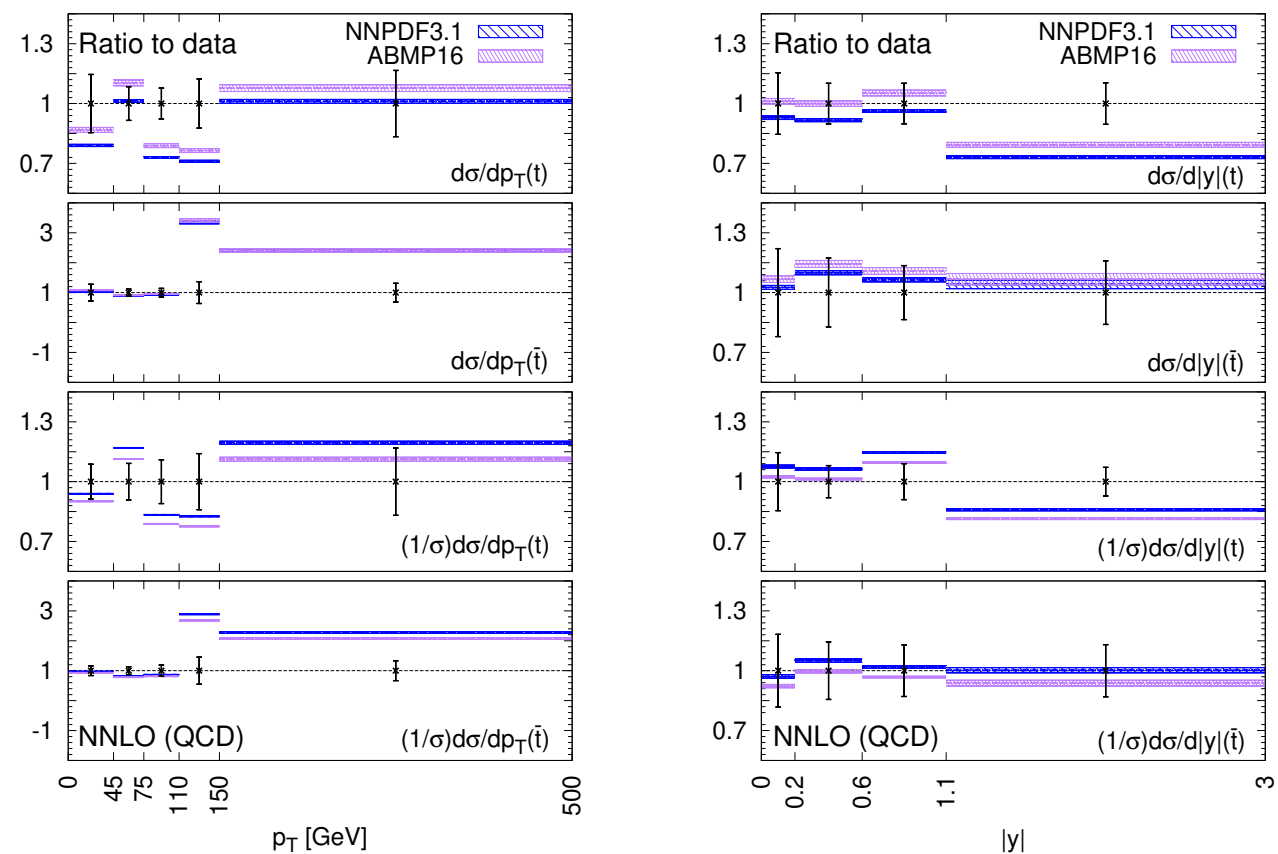

Figure 5. The $t$-channel absolute and normalised single top-(anti)quark distributions differential in the transverse momentum (left) and rapidity (right) of the top-(anti)quark (left). The measurements are compared to NNLO QCD theoretical predictions obtained either with the NNPDF31_nnlo_as_0118 or the ABMP16als118_5_nnlo PDF sets. The data and the theoretical predictions are normalised to the central value of the data.

\subsection{EW corrections}

Computations of NLO EW and NLO QCD $\times$ EW corrections to $t$-channel single top-quark and top-antiquark production were performed in refs. [27-29] in the context of supersymmetric extensions of the SM (a soft approximation to deal with real-emission contributions was employed in [27]). Corresponding Monte Carlo simulations have been implemented within the MADGRAPH5_AMC@NLO [57] (v3.0.1) generator, which we use to compute theoretical predictions accurate to $\mathrm{NLO} \mathrm{QCD} \times \mathrm{EW}$ for the data discussed in section 2 . To this purpose, we adopt the same physical parameters and settings as in section 3.1, and we take the PDFs (including the photon PDF) from the NNPDF31_nlo_as_0118_luxqed set [58].

Our results are compared to the NLO and NNLO QCD predictions in figure 2 for the ratio of the total top-quark to top-antiquark cross sections (or their sum, for the CMS data point at $7 \mathrm{TeV}$ ), and in figures $3-4$ for the top-quark and top-antiquark differential distributions, respectively. Results in figure 2 and in the lowest inset of figures 3-4 are normalised to the central value of the data points. Error bars (or bands) on theoretical predictions account for the $68 \%$ CL PDF uncertainty.

As is apparent from figures 2-4, EW corrections are almost negligible for the data under consideration here. For the ratios of single top-quark to top-antiquark total cross sections their effect cancels out completely; for their sum (in the case of the CMS data point at $7 \mathrm{TeV}$ ), they increase the NLO theoretical expectation by about $2 \%$, an amount 
which is comparable to the effect of the NNLO QCD correction, but still significantly smaller than the data and the PDF uncertainties. For the differential distributions, EW corrections account for a sub-percent effect, irrespective of whether the distributions are absolute or normalised, whether they are for top-quark or top-antiquark production, or whether they are differential in the transverse momentum or in the rapidity of the final particle. Such a result is consistent with what was found in ref. [59], where EW corrections larger than $1 \%$ were observed only for $p_{T}(t)$ and $p_{T}(\bar{t})$ bins above about $150 \mathrm{GeV}$, and at a centre-of-mass energy of $13 \mathrm{TeV}$. The size of such effects is much smaller than the statistical significance of the data, and obviously cannot explain the data-theory discrepancies observed in section 3.1 .

\subsection{Heavy flavour schemes}

The theoretical predictions presented so far were computed in the 5FS. This is a fixedflavour number (FFN) scheme whereby all quarks except the top quark are treated as massless partons. In this scheme, both the PDFs and the strong coupling evolve with five active flavours. The NNLO QCD corrections to $t$-channel single top production that we used to compute the $C$-factors in eq. (3.1) were obtained in this scheme. The process could otherwise be analysed in the 4FS [30], in which the bottom quark is produced at the matrixelement level and is not part of the proton wave-function. The NNLO QCD corrections for single top production in the $4 \mathrm{FS}$ are however not available, as the process starts at $\mathcal{O}\left(\alpha_{s}\right)$, and higher-order corrections including mass effects for the bottom quark are harder to compute. In this section, we investigate how predictions differ in the two schemes at $\mathrm{NLO}$, and study the effect of varying the bottom-quark matching point in the 5FS.

The 5FS is used as a default in all NNPDF determinations to describe a wide range of hadronic data; a matched general-mass variable flavour number (GM-VFN) scheme is used instead to describe deep-inelastic scattering (DIS) data [60]. The GM-VFN scheme adopted for DIS processes, namely the FONLL scheme, was originally devised for the transverse momentum spectrum of bottom quarks produced in hadronic collisions [61], and it was then generalised to DIS [62] and to the $b \bar{b} \rightarrow H[63,64]$ and $b \bar{b} \rightarrow Z$ [65] processes. In principle, since the FONLL scheme is universally applicable, it could easily be generalised to single top production to combine the $4 \mathrm{FS}$ and $5 \mathrm{FS}$ calculations performed at any perturbative order. Its availability would help overcome a drawback of the 5FS, namely that it neglects bottom-quark mass effects. In particular, mass corrections of $\mathcal{O}\left(m_{b}^{2} / Q^{2}\right)$, where $m_{b}$ is the bottom-quark mass, are set to zero above the scale at which the bottom PDF is activated. Mass corrections neglected in the 5FS are immaterial for most processes, except for those that depend on the bottom PDFs. Single top production, which is bottom-initiated at LO in the 5FS, is an example of such a process, and its theoretical description could therefore be affected by the choice of flavour scheme.

To assess the size of such effects, in table 3 we compare the predictions for inclusive single top production obtained at NLO in the 4FS and 5FS. The cross section is obtained by running MADGRAPH5_AMC@NLO with 4FS and 5FS settings, respectively. The NNPDF3.1 PDF input sets, NNPDF31_nlo_as_0118 and NNPDF31_nlo_as_0118_nf_4, have been determined in a consistent scheme. We compare predictions both for $\mu_{r}=\mu_{f} \equiv$ 


\begin{tabular}{|clll|}
\hline$\sqrt{s}[\mathrm{TeV}]$ & scale & $\sigma_{t}^{(5 \mathrm{FS})}[\mathrm{pb}]$ & $\sigma_{t}^{(4 \mathrm{FS})}[\mathrm{pb}]$ \\
\hline 7 & $\mu=m_{t}$ & $40.89{ }_{-2.3 \%}^{+3.2 \%} \pm 0.8 \%$ & $34.17_{-7.2 \%}^{+7.0 \%} \pm 0.8 \%$ \\
& $\mu=m_{t} / 4$ & $40.32_{-2.0 \%}^{+6.4 \%} \pm 0.8 \%$ & $38.37_{-5.1 \%}^{+4.1 \%} \pm 0.8 \%$ \\
\hline 8 & $\mu=m_{t}$ & $53.44_{-2.3 \%}^{+3.1 \%} \pm 0.8 \%$ & $45.17_{-6.8 \%}^{+6.5 \%} \pm 0.8 \%$ \\
& $\mu=m_{t} / 4$ & $53.08_{-2.7 \%}^{+5.9 \%} \pm 0.8 \%$ & $50.50{ }_{-4.8 \%}^{+4.0 \%} \pm 0.8 \%$ \\
\hline 13 & $\mu=m_{t}$ & $133.3_{-2.6 \%}^{+3.1 \%} \pm 0.8 \%$ & $115.9{ }_{-5.3 \%}^{+6.2 \%} \pm 0.8 \%$ \\
& $\mu=m_{t} / 4$ & $131.9_{-5.0 \%}^{+4.2 \%} \pm 0.8 \%$ & $126.2_{-3.9 \%}^{+3.3 \%} \pm 0.8 \%$ \\
\hline
\end{tabular}

Table 3. Predictions for single top production at NLO in the 5FS and the 4 FS. The 5FS (4FS) predictions have been computed using the corresponding NLO 5FS (4FS) input NNPDF3.1 PDF set. The first uncertainty quoted is the upper and lower missing higher-order uncertainty of the predictions, which is obtained by taking the envelope of the 7-point scale variation in which we vary the scales in the range $1 / 2 \leq \mu_{f}, \mu_{r}<2$. The second uncertainty represents the PDF uncertainty.

$\mu=m_{t}$ and for a lower scale $\mu_{r}=\mu_{f} \equiv \mu=m_{t} / 4$ motivated by the studies in [30,66]. While the PDF uncertainty is similar in both schemes, the missing higher-order uncertainty (MHOU) is larger in the 4FS than in the 5FS. Concerning predictions, the NLO total cross section in the $4 \mathrm{FS}$ is about $15 \%$ lower than the NLO total cross section in the 5FS. ${ }^{1}$ However, the difference between the predictions obtained in the two schemes, as well as between the size of the MHOUs, is considerably reduced when lower renormalisation and factorisation scales are used as central scale in the 4FS. The choice of such a scale is motivated by the following evidence: the scale that appears in the explicit collinear logarithms in the matrix element in the $m_{b} \rightarrow 0$ limit is proportional to the hard scale of the process, $m_{t}$ in this case, but is suppressed by a universal phase-space factor that is centered at about 0.25 at the LHC. Therefore the reduced difference between predictions, which amounts to about $5 \%$, is somewhat expected. The results are also displayed in figure 6 . The trend of the results is similar at 7,8 and $13 \mathrm{TeV}$.

In order to check the effect of the choice of scheme on the differential distributions, we display the data-theory comparison for the ATLAS absolute distributions predicted at NLO in either the $4 \mathrm{FS}$ or the $5 \mathrm{FS}$ for $\mu_{f}=\mu_{r}=m_{t}$, for which the difference between cross sections is larger. In the case of lower factorisation and renormalisation central scales, the differences are smaller, but similar features are exhibited. The results for $\mu_{f}=\mu_{r}=m_{t}$ are displayed in figure 7. As far as the overall normalisation is concerned, we notice that the 5FS predictions are closer to the data. As far as the shapes are concerned, they are quite similar for the rapidity distributions, while the two differ when the top quark has a small transverse momentum. This is not unexpected. As was noticed in the comparison of differential cross sections for charged Higgs boson production in ref. [67], the main

\footnotetext{
${ }^{1}$ The NLO corrections in the 4FS feature diagrams that belong to the NNLO corrections in the $5 \mathrm{FS}$. Therefore, one could compare the NNLO 5FS predictions to the NLO 4FS ones, in which case the difference would be slightly smaller, around $10 \%$.
} 


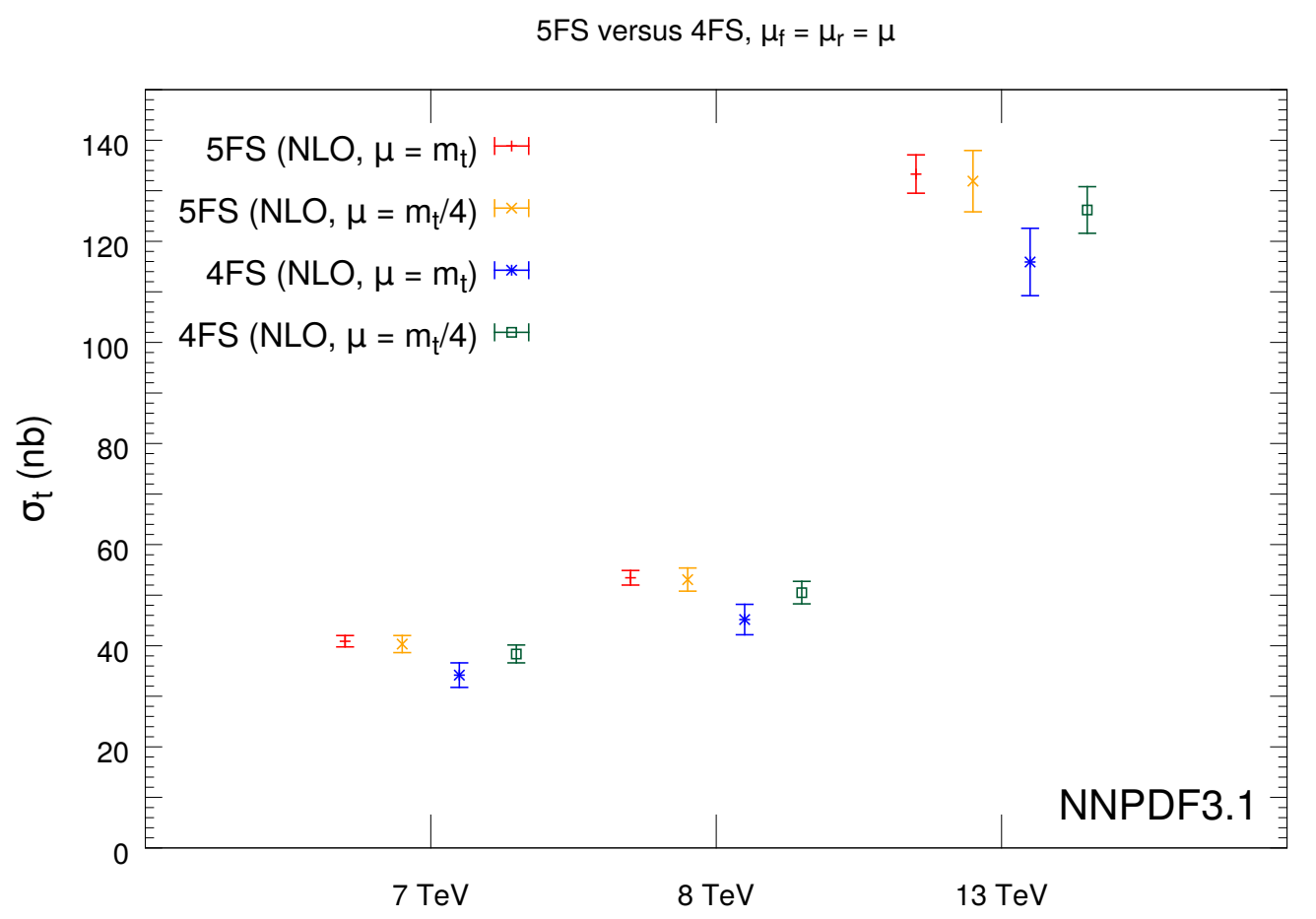

Figure 6. Comparison between total cross section predictions at NLO in the 5FS and in the 4FS for $\mu_{f}=\mu_{r}=m_{t}$ and for $\mu_{f}=\mu_{r}=m_{t} / 4$. The uncertainty of the theoretical predictions represent the envelope of 7 -point scale variations.

difference between the two schemes is the normalisation, as far as observables that are inclusive in the $b$-quark degrees of freedom are concerned. The top transverse momentum is more sensitive to the details of the $b$-quark transverse momentum at small $p_{T}$, while it is unaffected by it at large $p_{T}$. Clearly, once the transverse momentum of the $b$ quark is precisely measured and included in a fit of PDFs, the 4FS and 5FS descriptions would display larger shape differences and the 4FS would possibly be more adequate. However, an important conclusion that we can draw by looking at the plots in figure 7 is that the discrepancy between data and theory in the transverse momentum spectrum of the top and antitop at $7 \mathrm{TeV}$ is not due to the choice of scheme. This conclusion will be made quantitative in section 3.4.

Finally, we assess the impact of the bottom matching point, $\mu_{b}$, which is the scale at which the $b \mathrm{PDF}$ is activated in the 5FS. Conventionally this is set equal to the bottom mass, that is $\mu_{b}=m_{b}$, however there is no physical reason why this should be the case. In ref. [31] it was shown that the mass corrections neglected in the 5FS can be reduced by utilising the freedom one has in setting $\mu_{b}$. In particular, by increasing the bottom matching point in the range $m_{b}<\mu_{b} \leq 10 m_{b}$. A strong dependence of a given cross section on the choice of the matching point might stem from the fact that matching conditions are only known to the lowest nontrivial order. As far as charm-initiated processes are concerned, it was shown that it is advantageous to treat the charm PDF on the same footing as lightquark PDFs; that is, to parametrise it and extract it from data, rather than to take it 

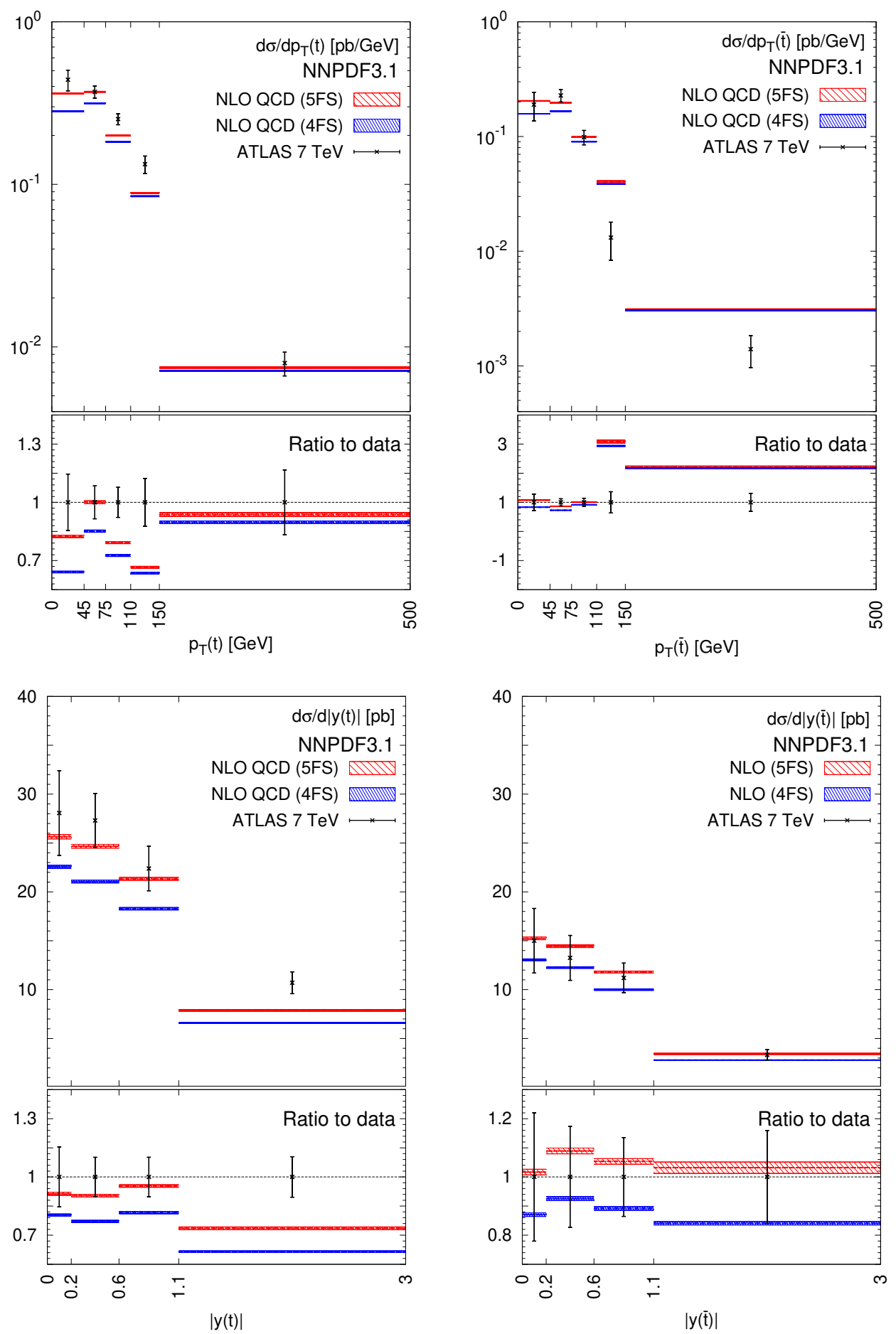

Figure 7. Same as figure 3, but comparing NLO predictions in the 4FS and 5FS. 
as radiatively generated through perturbative matching conditions [68-70]. In ref. [71] a similar study was performed in the context of the bottom $b$ PDF and the FONLL scheme.

Here we consider three NLO PDF sets obtained by varying the bottom matching points as follows: $\mu_{b}=m_{b} ; \mu_{b}=2 m_{b}$; and $\mu_{b}=5 m_{b}$. The PDF set with $\mu_{b}=m_{b}$ coincides with the standard NNPDF3.1 PDF set with $\alpha_{s}\left(M_{Z}\right)=0.118$. The PDFs are evolved from the initial scale $Q_{0}$ up to the scale $\mu_{f}=m_{t}$, at which we compute the single top total cross section, by crossing the bottom matching point $\mu_{b}$. Results are displayed in figure 8. We notice that the dependence of the single top cross section on the bottomquark matching point is quite strong, with predictions decreasing by $5-7 \%(10-12 \%)$ as the bottom matching point is raised to twice (five times) the mass of the bottom quark. Furthermore, as $\mu_{b}$ increases, the 5FS predictions get closer to the $4 \mathrm{FS}$ ones. We have checked that, using the NNLO PDF sets obtained by varying the bottom matching point, the NLO predictions still decrease but the effect is much smaller, of order $2 \%(3-4 \%)$ as the bottom matching point is raised to twice (five times) the mass of the bottom quark. To fully assess this effect at NNLO, we would need to have the NNLO calculations obtained with higher bottom matching points. These results suggest that the choice of a bottom matching point higher than $m_{b}$ has a non negligible effect on the theoretical predictions, whose size is comparable to that of missing power corrections of $m_{b}$ in a 5FS. Such effects are well below the experimental uncertainty of the data discussed here. However, when more precise data become available, it will be crucial to either perform dedicated studies to determine the optimal bottom matching point $\mu_{b}$ that minimises missing power corrections of $m_{b}$ in a 5FS, or to explicitly compute matched predictions à la FONLL for single top observables, in order to directly include $b$-quark mass corrections via the matching of the $5 \mathrm{FS}$ to the $4 \mathrm{FS}$ cross sections.

\subsection{Quantitative assessment of the agreement between theory and data}

A quantitative assessment of the agreement between theoretical expectations and the data presented above requires an appropriate statistical measure, which should take into account experimental correlations. We therefore define the $\chi^{2}$ per data point (called $\chi^{2}$ henceforth)

$$
\chi^{2}\{\mathcal{T}[f], \mathcal{D}\}=\frac{1}{N_{\text {dat }}} \sum_{i, j}^{N_{\text {dat }}}\left(T_{i}[f]-D_{i}\right) C_{i j}^{-1}\left(T_{j}[f]-D_{j}\right),
$$

which depends on the dataset, $\mathcal{D}$, and on the theoretical predictions computed with the PDFs $f, \mathcal{T}[f]$. The indices $i$ and $j$ run over the experimental data points, $D_{i}$ are their measured central values, $T_{i}$ are the corresponding theoretical predictions (computed with a given theory and a given set of PDFs), and $C_{i j}$ is the covariance matrix, constructed from the available information on experimental statistical and systematic uncertainties. In eq. (3.2), we specifically use the experimental covariance matrix

$$
C_{i j}=C_{i j}^{\mathrm{exp}} \equiv \delta_{i j}\left(s_{i}^{\mathrm{stat}}\right)^{2}+\left[\sum_{\alpha=1}^{N_{\mathrm{sysA}}} \sigma_{i, \alpha}^{\mathrm{sysA}} \sigma_{j, \alpha}^{\mathrm{sys}}+\sum_{\beta=1}^{N_{\mathrm{sysM}}} \sigma_{i, \beta}^{\mathrm{sysM}} \sigma_{j, \beta}^{\mathrm{sysM}}\right] D_{i} D_{j}
$$


5FS versus $4 F S$ at NLO, varying $\mu_{b}, \mu_{f}=\mu_{r}=m_{t}$

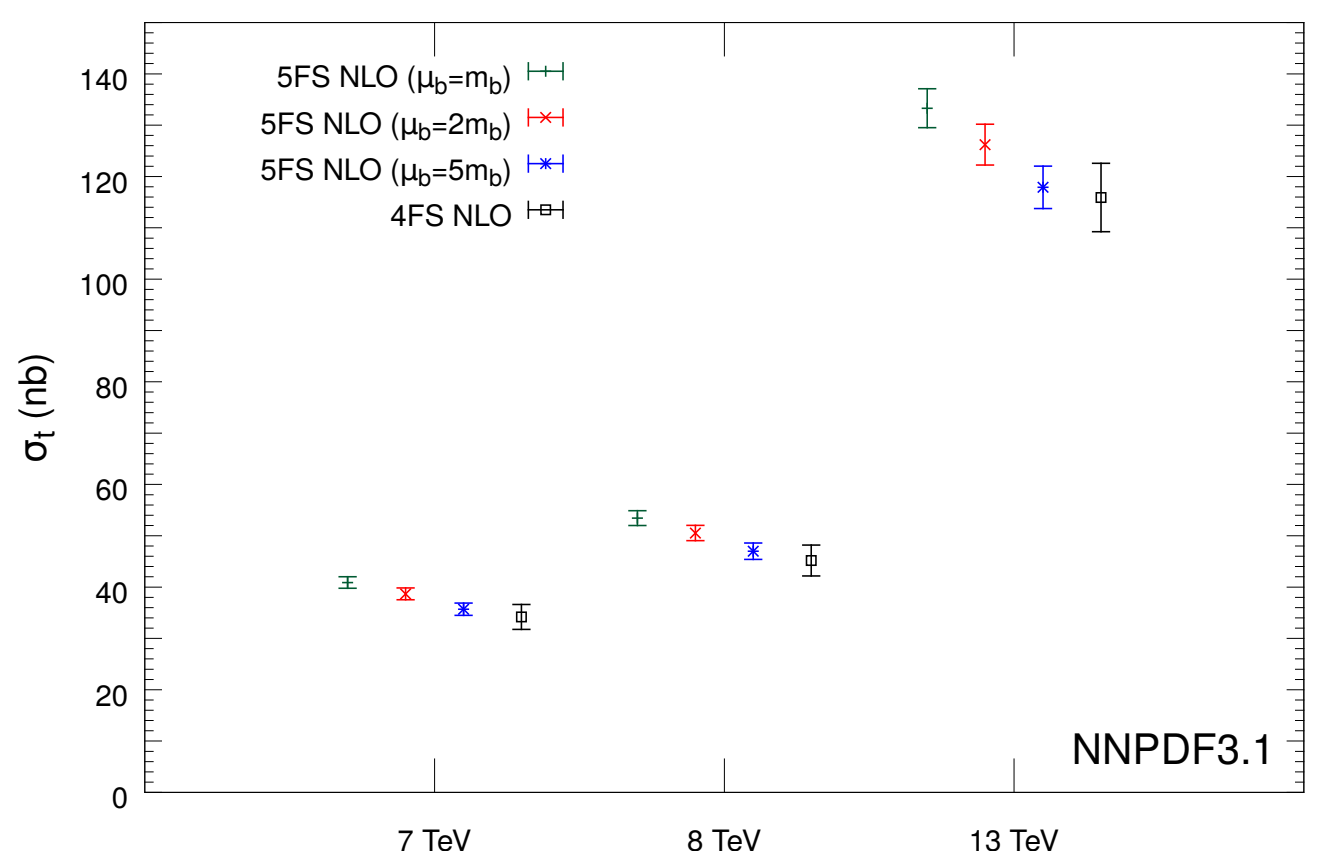

Figure 8. Comparison between 4FS and 5FS NLO predictions for $\mu_{f}=\mu_{r}=m_{t}$ and varying $\mu_{b}$. We display predictions at NLO for $\mu_{b}=m_{b}, 2 m_{b}$ and $5 m_{b}$, and compare them to the 4FS NLO predictions.

where $s_{i}^{\text {stat }}$ is the uncorrelated absolute uncertainty of the data point (obtained by adding in quadrature statistical and uncorrelated systematic uncertainties $)$, and $\sigma_{i}^{\text {sysA }}\left(\sigma_{i}^{\text {sysM }}\right)$ are the $N_{\text {sysA }}\left(N_{\text {sysM }}\right)$ correlated additive (multiplicative) relative systematic uncertainties.

The values of the $\chi^{2}$ computed using eq. (3.3) are collected in table 4 for all of the datasets presented in tables 1-2. The last bin of the normalised distributions is excluded from the computation. They are presented for predictions obtained within three different theoretical scenarios: NLO and NNLO in the 5FS and NLO in the 4FS. The input PDF sets are chosen consistently: NNPDF31_nlo_as_0118, NNPDF31_nnlo_as_0118 and NNPDF31_nlo_as_0118_nf_4, respectively. In the case of NNLO QCD within the 5FS, we also report the values of the $\chi^{2}$ obtained with the ABMP16als118_5_nnlo PDF set, to complete the data-theory comparisons displayed in figures $2-5$. We have explicitly checked that $\chi^{2}$ variations induced by the use of other PDF sets, such as MMHT [52], CT18 [53], or the PDF4LHC15 combination [54], and theoretical expectations accurate to NLO QCD $\times$ EW (computed with the NNPDF31_nlo_as_0118_luxqed PDF set) are tiny, and do not alter the interpretation of these results. The values of the $\chi^{2}$ obtained upon these variations are therefore not shown in table 4 . In order to assess the impact of correlations in the 5FS, we report in parentheses the $\chi^{2}$ values computed by neglecting bin-by-bin correlations.

The results collected in table 4 quantitatively confirm the pattern of data-theory inconsistencies already qualitatively displayed in figures $2-5$. While most of the data are fairly well described by the theoretical predictions, irrespective of their accuracy, there are 


\begin{tabular}{|lcccllll|}
\hline \multirow{2}{*}{ Experiment } & $\sqrt{s}[\mathrm{TeV}]$ & $\mathcal{O}$ & $N_{\mathrm{dat}}$ & $\chi_{\mathrm{NLO}}^{2(5 \mathrm{FS})}$ & $\chi_{\mathrm{NNLO}}^{2(5 \mathrm{FS})}$ & $\chi_{\mathrm{NLO}}^{2(4 \mathrm{FS})}$ & $\chi_{\mathrm{NNLO}, \mathrm{ABMP} 16}^{2(5 \mathrm{FS})}$ \\
\hline ATLAS & 7 & $\sigma_{t} / \sigma_{\bar{t}}$ & 1 & $0.28(-)$ & $0.30(-)$ & 0.41 & 0.42 \\
& 8 & $\sigma_{t} / \sigma_{\bar{t}}$ & 1 & $3.71(-)$ & $3.67(-)$ & 3.06 & 2.69 \\
& 13 & $\sigma_{t} / \sigma_{\bar{t}}$ & 1 & $0.03(-)$ & $0.03(-)$ & 0.02 & 0.13 \\
\hline CMS & 7 & $\sigma_{t+\bar{t}}$ & 1 & $0.82(-)$ & $0.66(-)$ & 6.28 & 0.02 \\
& 8 & $\sigma_{t} / \sigma_{\bar{t}}$ & 1 & $0.09(-)$ & $0.09(-)$ & 0.14 & 0.17 \\
& 13 & $\sigma_{t} / \sigma_{\bar{t}}$ & 1 & $0.29(-)$ & $0.29(-)$ & 0.25 & 0.48 \\
\hline ATLAS & 7 & $d \sigma / d p_{T}(t)$ & 5 & $2.16(1.24)$ & $2.88(1.45)$ & 2.22 & 3.15 \\
& & $d \sigma / d p_{T}(\bar{t})$ & 5 & $9.49(3.31)$ & $11.9(4.15)$ & 12.4 & 11.5 \\
& & $d \sigma / d|y(t)|$ & 4 & $1.16(0.97)$ & $1.29(0.93)$ & 1.99 & 1.36 \\
& & $d \sigma / d|y(\bar{t})|$ & 4 & $0.05(0.07)$ & $0.06(0.08)$ & 0.13 & 0.11 \\
& & $(1 / \sigma) d \sigma / d p_{T}(t)$ & 4 & $1.89(0.99)$ & $2.79(1.29)$ & 1.76 & 2.32 \\
& $(1 / \sigma) d \sigma / d p_{T}(\bar{t})$ & 4 & $5.14(2.76)$ & $6.26(3.34)$ & 7.14 & 6.81 \\
& $(1 / \sigma) d \sigma / d|y(t)|$ & 3 & $0.92(1.11)$ & $0.96(1.18)$ & 1.19 & 2.13 \\
& & $(1 / \sigma) d \sigma / d|y(\bar{t})|$ & 3 & $0.07(0.04)$ & $0.07(0.04)$ & 0.05 & 1.36 \\
\hline
\end{tabular}

Table 4. The values of the $\chi^{2}$, eq. (3.2), computed for all of the datasets described in section 2 and collected in tables 1-2. For ease of reference, we indicate the centre-of-mass energy of each measurement, $\sqrt{s}$, the corresponding observable, $\mathcal{O}$, and the number of data points, $N_{\text {dat }}$. Theoretical predictions are computed at either NLO or NNLO accuracy in the 5FS or at NLO accuracy in the $4 \mathrm{FS}$, always in pure QCD; the input PDFs are taken from the NNPDF31_nlo_as_0118, NNPDF31_nnlo_as_0118 and NNPDF31_nlo_as_0118_nf_4 sets, respectively. Values in parentheses do not include bin-by-bin experimental correlations.

three notable exceptions. The first is the ratio of single top-quark to top-antiquark total cross sections from ATLAS at $8 \mathrm{TeV}\left(\chi^{2} \sim 3-4\right)$; the second is the distribution differential in the top-antiquark transverse momentum $p_{T}(\bar{t})\left(\chi^{2} \sim 10-12\right)$; and the third is the corresponding normalised distribution $\left(\chi^{2} \sim 6\right)$. In the first case, we note that the analogous measurement from CMS, which differs from the ATLAS one by approximately $\sqrt{2} \sigma$, is instead very well described. We therefore conclude that the two points might be somewhat in tension, for reasons that do not seem to depend on the accuracy of the theoretical predictions. In the second and third cases, the high $\chi^{2}$ values are a consequence of the large data-theory discrepancy (up to 300\%) observed in the fourth and fifth transverse momentum bins. Despite a good $\chi^{2}$ being reported for this distribution in ref. [12] at NLO (see table VIII therein), also in these cases, we are not able to envision theoretical effects that could explain such a difference (nor the $\chi^{2}$ values reported in ref. [12]), as we further discuss below.

Overall, NNLO QCD corrections have a moderate impact. In the case of ratios of single top-quark to top-antiquark total cross sections their effect on the $\chi^{2}$ cancels out almost completely; for their sum (in the case of the CMS dataset at $7 \mathrm{TeV}$ ), they instead tend to improve the description of the data point a little (the $\chi^{2}$ decreases from 0.82 to 0.66). In the case of differential distributions, NNLO QCD corrections generally lead to a worsening of the $\chi^{2}$, which is significant in particular for the distributions differential in the transverse momentum of the top quark or top antiquark. 
The $4 \mathrm{FS}$ leads to a worse $\chi^{2}$ value than the $5 \mathrm{FS}$ for most of the datasets, consistently with the qualitative behaviour observed in figure 7 . However, while the increase in the $\chi^{2}$ can be substantial for the sum of top-quark and top-antiquark cross sections, it is moderate for the differential distributions and only barely noticeable for their ratio: the 4FS modifies the individual cross sections in such a way that their variations cancel out in the ratio.

More strikingly, experimental correlations have a large effect: neglecting them leads to an acceptable $\chi^{2}$ for all of the differential distributions, except, again, for those differential in the transverse momentum of the top-antiquark $p_{T}(\bar{t})$ (for which the $\chi^{2}$ improvement is nevertheless the largest).

This state of affairs leads us to wonder whether the poor description of some single top-quark and top-antiquark datasets follows from internal inconsistencies or from inconsistencies with the rest of the dataset. As far as the ATLAS differential distributions at $\sqrt{s}=7 \mathrm{TeV}$ are concerned, we hypothesise that they are internally inconsistent. Our hypothesis is driven by the fact that the data-theory discrepancies persist no matter what theoretical framework or PDF set we use. Furthermore, we also investigate how other single top-quark and top-antiquark datasets, not considered in this section, are described. We do so with the ATLAS differential measurements at a centre-of-mass energy of $8 \mathrm{TeV}$ [13] in appendix A. Even though no information on correlations is available for this dataset, as explained in section 2, we do not observe any significant data-theory discrepancy.

Of course, only the inclusion of the $7 \mathrm{TeV}$ data in a PDF fit will tell us whether they are inconsistent with the rest of the dataset, and if the observed discrepancies can be resolved by altering the shape of the PDFs (see section 4). To include the data presented in tables 12 in a global fit, we shall decide which theoretical framework is the most convenient for their analysis. Because one of our goals is to assess the interplay of single top-quark and topantiquark data with the rest of the dataset, NNLO corrections are mandatory to achieve the best overall description we can. However, the use of NNLO QCD forces us to the 5FS, as higher-order corrections to single top-quark and top-antiquark data are not known in the 4 FS. Theoretical predictions will therefore be computed accordingly in section 4 .

We shall however note that the experimental covariance matrix defined in eq. (3.3), used in this section, is not suitable for PDF fits. The reason being that it would lead to a fit that systematically undershoots the data: this is the so-called D'Agostini bias [72], which is related to an inappropriate treatment of multiplicative uncertainties. We therefore define an alternative covariance matrix that removes such a bias

$$
C_{i j}=C_{i j}^{t_{0}} \equiv \delta_{i j}\left(s_{i}^{\mathrm{stat}}\right)^{2}+\left(\sum_{\alpha}^{N_{\mathrm{sysA}}} \sigma_{i, \alpha}^{\mathrm{sys}} \sigma_{j, \alpha}^{\mathrm{sysA}}\right) D_{i} D_{j}+\left(\sum_{\beta}^{N_{\mathrm{sysB}}} \sigma_{i, \beta}^{\mathrm{sysB}} \sigma_{j, \beta}^{\mathrm{sysB}}\right) T_{i}^{(0)} T_{j}^{(0)},
$$

where multiplicative uncertainties are multiplied by the theoretical predictions $\left\{T_{i}^{(0)}\right\}$. This is the $t_{0}$-prescription [73], which we use in all of the PDF fits presented in section 4 .

\section{Impact of single top data on PDFs}

In this section we assess the impact on PDFs of the single top data discussed previously. Firstly, we study the correlations between the data and PDFs. Then we outline the settings 
of our PDF fits, and we detail the baseline dataset on top of which we add single top data. Next, the results of the PDF fits are presented. We then study the correlations between single top data and other measurements used in the fits, before finishing with a discussion of the impact of single top data on LHC phenomenology.

\subsection{Observable-PDF correlations}

Before assessing the impact of single top data in a PDF fit, it can be instructive to compute the correlation coefficient $\rho[74]$ between the PDFs and the single top data. We calculate $\rho$ between a given observable $\mathcal{O}$ and a PDF $f$ as

$$
\rho(\mathcal{O}, f)=\frac{\langle\mathcal{O} f\rangle-\langle\mathcal{O}\rangle\langle f\rangle}{\Delta_{\mathcal{O}} \Delta_{f}}
$$

where the angled brackets denote the mean and $\Delta$ denotes the uncertainty on $\mathcal{O}$ or $f$, both computed over an ensemble of PDF replicas. For fixed energy scale $Q$ and Bjorken- $x, \rho$ is a number between -1 and 1 , with its magnitude indicating the degree to which $\mathcal{O}$ is sensitive to $f$. If the single top data have any impact on $f$ in a PDF fit, then the regions of high correlation are the regions where we would expect to see changes in $f$. Note that these correlations are inherently dependent on the data that are already included in the fit of the PDFs $f$. That is, the correlations depend on the way in which single top production is initiated at the parton level and the kinematics of single top production, but also on the experimental constraints included in the determination of $f$. This means that the maxima of $|\rho|$ are in general not solely dependent on what the most favoured initial state is, and in which kinematic region this dependence is most prominent. As a result, the maxima are likely to be displaced from the values that one might naively expect.

In what follows we restrict our analysis to the up-quark $(u)$, the down-quark $(d)$ and the gluon $(g)$ PDFs, since these are three of the main partons that initiate single top production. Note that we ignore the up antiquark $(\bar{u})$ and the down antiquark $(\bar{d})$ here because the sensitivity of the single top data to them can largely be inferred from the behaviour of the $u$ and the $d$. We compute $\rho$ using the NNPDF31_nnlo_as_0118 PDF set at an energy scale equal to the top mass, i.e. $Q=m_{t}=172.5 \mathrm{GeV}$.

We begin by looking at the inclusive total cross section measurements, $\sigma_{t}+\sigma_{\bar{t}}$, and at the ratio $\sigma_{t} / \sigma_{\bar{t}}$. In refs. [10-12] it has been suggested that measurements of $\sigma_{t} / \sigma_{\bar{t}}$ could be used to constrain the ratio $u / d$ in the region $0.02 \lesssim x \lesssim 0.5$. This has been stated as a reason in favour of using measurements of $\sigma_{t} / \sigma_{\bar{t}}$ in PDF determinations, in addition to the fact that common systematic uncertainties are likely to at least partially cancel when the ratio is taken, which makes these measurements comparatively precise [12-14].

Figure 9 displays two representative plots of the correlation coefficient as a function of $x$. The left panel shows the correlation between $u / d$ and the ATLAS measurement of $\sigma_{t} / \sigma_{\bar{t}}$ at $13 \mathrm{TeV}$, while the right panel shows the correlation between $g$ and the CMS measurement of $\sigma_{t}+\sigma_{\bar{t}}$ at $7 \mathrm{TeV}$. We see that for the ATLAS ratio measurement the maximum correlation is observed for $x \lesssim 10^{-3}$, with $\rho$ peaking at around 0.7 for $x=10^{-4}$. The same general behaviour is observed for each of the five $\sigma_{t} / \sigma_{\bar{t}}$ measurements that are detailed in section 2 , and is therefore not explicitly displayed in figure 9 . In fact, the same characteristic minima 

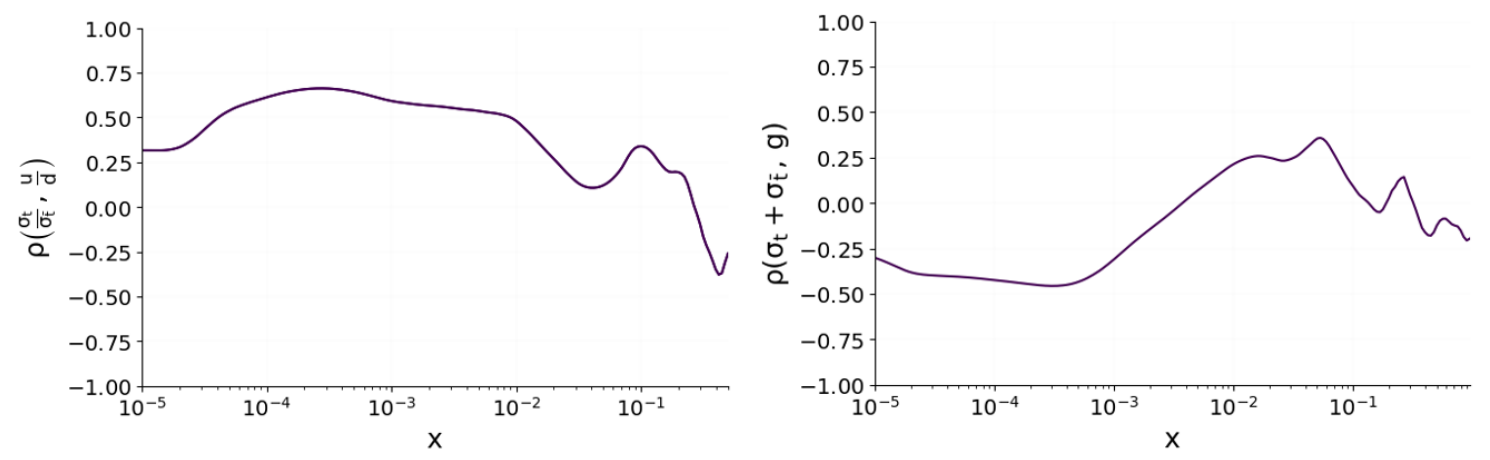

Figure 9. Correlation coefficient $\rho$ between the up-quark/down-quark PDF ratio and the ATLAS measurement of $\sigma_{t} / \sigma_{\bar{t}}$ at $13 \mathrm{TeV}$ (left panel), and the gluon and the CMS measurement of $\sigma_{t}+\sigma_{\bar{t}}$ at $7 \mathrm{TeV}$ (right panel). The correlation coefficient $\rho$ is computed using the NNPDF31_nnlo_as_0118 PDF set at $Q=m_{t}=172.5 \mathrm{GeV}$. Note that the $x$-axis in the left panel runs between $x=10^{-5}$ and $x=0.5$. This truncation of large values of $x$ is due to the fact that as $x \rightarrow 1, u / d \rightarrow 0 / 0$, which leads to numerical instabilities in $\rho$.

and maxima are seen for each ratio measurement, with two maxima observed between $x=0.1$ and $x=0.2$ and a region of maximum anti-correlation observed at $x \simeq 0.4$. For the corresponding measurements at lower values of $\sqrt{s}$, the sensitivity weakens slightly as $\sqrt{s}$ decreases. These observations back up the claim made in ref. [12] that measurements of $\sigma_{t} / \sigma_{\bar{t}}$ are sensitive to $u / d$, although the highest sensitivity is seen at lower values of $x$ than was originally suggested. We find that, in contrast, these measurements are mostly insensitive to the gluon PDF.

In comparison, the right panel of figure 9 shows that the inclusive total cross section $\sigma_{t}+\sigma_{\bar{t}}$ is somewhat sensitive to the gluon, in particular around $x \simeq 5 \times 10^{-4}(\rho \simeq-0.5)$ and $x \simeq 0.05(\rho \simeq 0.5)$. However, while $\sigma_{t}+\sigma_{\bar{t}}$ is more sensitive to the gluon, it is less sensitive to the $u$ and $d$ - and it has very low sensitivity to $u / d$ - as we have explicitly checked by looking at the corresponding values of $\rho$. We therefore conclude that the gluon sensitivity largely cancels when the top-quark and top-antiquark cross sections are divided, as in $\sigma_{t} / \sigma_{\bar{t}}$, but not when they are summed, as in $\sigma_{t}+\sigma_{\bar{t}}$. The reverse is true for $u / d$. This is because $\sigma_{t}+\sigma_{\bar{t}}$ contains no information on the relative strength of the $u$ and $d$ contributions to the process, while $\sigma_{t} / \sigma_{\bar{t}}$ does.

It might at first appear surprising that $\sigma_{t}+\sigma_{\bar{t}}$ is sensitive to the gluon, since at LO in the 5FS single top production is not initiated by the gluon. However, firstly, this is not true at NLO in the 5FS, and secondly, single top production at LO in the 5FS is initiated by the bottom quark, which is generated perturbatively from the gluon PDF. Therefore, single top production is in general sensitive to the gluon, even at LO.

We now look at the correlation coefficient between the PDFs and bins of the single top differential distributions. Figure 10 shows the correlation coefficient between the gluon and two single top distributions. The left panel is for the ATLAS $7 \mathrm{TeV}$ measurement of the transverse momentum of the top quark, while the right panel is for the ATLAS $7 \mathrm{TeV}$ single top measurement of the rapidity of the top quark. Both distributions are absolute. 

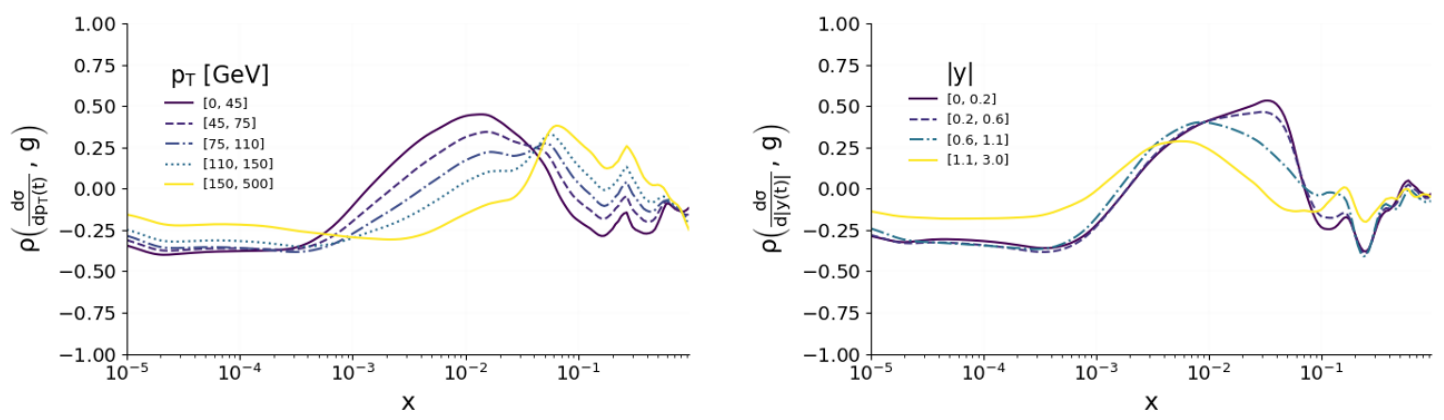

Figure 10. Same as figure 9 but for the absolute ATLAS $7 \mathrm{TeV}$ differential measurement of the topquark transverse momentum (left panel) and the absolute ATLAS $7 \mathrm{TeV}$ differential measurement of the top-quark rapidity (right panel). Both panels show the correlation coefficient for the gluon. The items in the legend indicate the bins that each of the lines corresponds to.

For the $p_{T}(t)$ distribution, we observe that each of its bins is sensitive to the gluon, but that the shape of $\rho$ is somewhat bin-dependent. The largest correlation $(\rho \simeq 0.5)$ is seen around $x \simeq 10^{-2}$, in particular for the lowest $p_{T}$ bin. For the $|y(t)|$ distribution, we see that it displays a correlation with the gluon PDF similar to the $p_{T}(t)$ distribution. The lowest rapidity bins are most sensitive to the gluon, as they display a correlation peak $(\rho \simeq 0.5)$ at $x \simeq 0.03$.

We systematically looked at the correlations between the $p_{T}(t)$ and $|y(t)|$ distributions and the $u$ and $d$ PDFs, as well as at the correlations between the top-antiquark distributions and all partons. Such correlations generally appear to be weaker than those displayed in figure 10, and are therefore not explicitly shown. The only notable exceptions are, as expected, correlations of about $\rho \simeq 0.5$ between the $u(d)$ and the $p_{T}(t)$ distribution around $x \simeq 0.5(x \simeq 0.6)$. In this region, the higher the transverse momentum of the bin, the higher the sensitivity. The correlation pattern between the gluon and any of the top-antiquark distributions is similar to the one displayed in figure 10.

\section{2 $\quad$ Fit settings}

Having assessed the sensitivity of PDFs to the single top data presented in section 2, we can now study whether this sensitivity leads to any change in the PDFs. We do so by including the data in a PDF fit on top of a baseline dataset. Specifically, we consider the NNPDF3.1 dataset of ref. [75]. This includes fixed-target neutral-current (NC) DIS structure function data from NMC [76, 77], SLAC [78] and BCDMS [79, 80]; charged-current (CC) DIS structure function data from CHORUS [81] and $\mathrm{NuTeV}$ [82, 83]; HERA measurements from their combined datasets [84], including charm-production cross sections [85] and $b$ tagged structure functions [86, 87]; fixed-target Drell-Yan data from E866 [88-90] and E605 [91]; Tevatron data from CDF [92] and D0 [93-95]; and LHC data from ATLAS [96104], CMS [105-113] and LHCb [114-117]. In total this baseline dataset contains $N_{\text {dat }}=$ 3979 data points.

To avoid double counting, the single top data considered here cannot be all included at the same time (see section 2). We therefore perform a series of fits, where various 


\begin{tabular}{|lcccccc|}
\hline Dataset & Fit 1 & Fit 2 & Fit 3 & Fit 4 & Fit 5 & Optimal fit \\
\hline Global baseline & $\mathbf{y}$ & $\mathbf{y}$ & $\mathbf{y}$ & $\mathbf{y}$ & $\mathbf{y}$ & $\mathbf{y}$ \\
\hline ATLAS $\sigma_{t} / \sigma_{\bar{t}} 7 \mathrm{TeV}$ & $\mathrm{n}$ & $\mathrm{n}$ & $\mathrm{n}$ & $\mathbf{y}$ & $\mathbf{y}$ & $\mathbf{y}$ \\
ATLAS $\sigma_{t} / \sigma_{\bar{t}} 8 \mathrm{TeV}$ & $\mathrm{n}$ & $\mathbf{y}$ & $\mathbf{y}$ & $\mathbf{y}$ & $\mathbf{y}$ & $\mathrm{n}$ \\
ATLAS $\sigma_{t} / \sigma_{\bar{t}} 13 \mathrm{TeV}$ & $\mathrm{n}$ & $\mathbf{y}$ & $\mathbf{y}$ & $\mathbf{y}$ & $\mathbf{y}$ & $\mathbf{y}$ \\
CMS $\sigma_{t} / \sigma_{\bar{t}} 8 \mathrm{TeV}$ & $\mathrm{n}$ & $\mathbf{y}$ & $\mathbf{y}$ & $\mathbf{y}$ & $\mathbf{y}$ & $\mathbf{y}$ \\
CMS $\sigma_{t} / \sigma_{\bar{t}} 13 \mathrm{TeV}$ & $\mathrm{n}$ & $\mathbf{y}$ & $\mathbf{y}$ & $\mathbf{y}$ & $\mathbf{y}$ & $\mathbf{y}$ \\
CMS $\sigma_{t+\bar{t}} 7 \mathrm{TeV}$ & $\mathrm{n}$ & $\mathbf{y}$ & $\mathbf{y}$ & $\mathbf{y}$ & $\mathbf{y}$ & $\mathbf{y}$ \\
ATLAS $d \sigma / d p_{T}(t) 7 \mathrm{TeV}$ & $\mathrm{n}$ & $\mathbf{y}$ & $\mathrm{n}$ & $\mathrm{n}$ & $\mathrm{n}$ & $\mathrm{n}$ \\
ATLAS $d \sigma / d p_{T}(\bar{t}) 7 \mathrm{TeV}$ & $\mathrm{n}$ & $\mathbf{y}$ & $\mathrm{n}$ & $\mathrm{n}$ & $\mathrm{n}$ & $\mathrm{n}$ \\
ATLAS $d \sigma / d|y(t)| 7 \mathrm{TeV}$ & $\mathrm{n}$ & $\mathrm{n}$ & $\mathbf{y}$ & $\mathrm{n}$ & $\mathrm{n}$ & $\mathrm{n}$ \\
ATLAS $d \sigma / d|y(\bar{t})| 7 \mathrm{TeV}$ & $\mathrm{n}$ & $\mathrm{n}$ & $\mathbf{y}$ & $\mathrm{n}$ & $\mathrm{n}$ & $\mathrm{n}$ \\
ATLAS $(1 / \sigma) d \sigma / d p_{T}(t) 7 \mathrm{TeV}$ & $\mathrm{n}$ & $\mathrm{n}$ & $\mathrm{n}$ & $\mathbf{y}$ & $\mathrm{n}$ & $\mathrm{n}$ \\
ATLAS $(1 / \sigma) d \sigma / d p_{T}(\bar{t}) 7 \mathrm{TeV}$ & $\mathrm{n}$ & $\mathrm{n}$ & $\mathrm{n}$ & $\mathbf{y}$ & $\mathrm{n}$ & $\mathrm{n}$ \\
ATLAS $(1 / \sigma) d \sigma / d|y(t)| 7 \mathrm{TeV}$ & $\mathrm{n}$ & $\mathrm{n}$ & $\mathrm{n}$ & $\mathrm{n}$ & $\mathbf{y}$ & $\mathbf{y}$ \\
ATLAS $(1 / \sigma) d \sigma / d|y(\bar{t})| 7 \mathrm{TeV}$ & $\mathrm{n}$ & $\mathrm{n}$ & $\mathrm{n}$ & $\mathrm{n}$ & $\mathbf{y}$ & $\mathbf{y}$ \\
\hline
\end{tabular}

Table 5. The combinations of data that are included (y) or not (n) in each fit.

combinations of single top measurements are added on top of the baseline dataset outlined above. These combinations, which define Fit $2-$ Fit 5 , are summarised in table 5. A fit without any single top data (Fit 1) and a fit containing the optimal choice of single top measurements according to our investigations below (Optimal fit) are performed as reference. All fits are iterated to reach convergence of the $t_{0}$-method (see section 3.4).

The realisation of the fits otherwise closely follows the NNPDF3.1 methodology [32]. In this analysis, we use $\alpha_{s}\left(M_{Z}\right)=0.118$, in line with the current recommended value from the PDG [118]. The charm-quark, bottom-quark and top-quark pole masses are set as in the NNPDF3.1 default analysis to $m_{c}=1.51 \mathrm{GeV}, m_{b}=4.92 \mathrm{GeV}$ and $m_{t}=$ $172.5 \mathrm{GeV}$, respectively, in accordance with the values recommended by the Higgs Cross Section Working Group [119]. The PDFs are parametrised and fitted at $Q_{0}=1.65 \mathrm{GeV}$ and then evolved at NNLO with APFEL [120]. The REPORTEnGine software [121] is used to analyse each fit.

\subsection{Fit results}

We now present the results of the fits summarised in table 5 . Table 6 shows the $\chi^{2}$ values for the datasets included in the various fits, computed according to eqs. (3.2)-(3.3). Numbers in boldface (square brackets) denote the single top measurements (not) included in each fit. The Baseline fit has a $\chi^{2}=1.19$ which remains stable upon the inclusion of single top data in the fit, irrespective of the single top distribution considered.

Turning our attention to the results for individual single top datasets, we observe, overall, a remarkable stability in the values of the $\chi^{2}$ before and after the fit, irrespective 


\begin{tabular}{|c|c|c|c|c|c|c|c|c|}
\hline Dataset & Ref. & $N_{\text {dat }}$ & Fit 1 & Fit 2 & Fit 3 & Fit 4 & Fit 5 & Optimal fit \\
\hline NMC & {$[76,77]$} & 325 & 1.31 & 1.31 & 1.32 & 1.31 & 1.30 & 1.31 \\
\hline SLAC & {$[78]$} & 67 & 0.76 & 0.75 & 0.76 & 0.78 & 0.75 & 0.75 \\
\hline BCDMS & {$[79,80]$} & 581 & 1.18 & 1.18 & 1.19 & 1.19 & 1.19 & 1.19 \\
\hline CHORUS & {$[81]$} & 832 & 1.13 & 1.12 & 1.13 & 1.13 & 1.13 & 1.12 \\
\hline NuTeV & {$[82,83]$} & 76 & 0.83 & 0.81 & 0.86 & 0.81 & 0.85 & 0.87 \\
\hline HERA $\sigma_{\mathrm{NC}, \mathrm{CC}}^{p}$ & {$[84]$} & 1145 & 1.16 & 1.16 & 1.15 & 1.15 & 1.16 & 1.16 \\
\hline HERA $\sigma_{\mathrm{NC}}^{c}$ & {$[85]$} & 37 & 1.47 & 1.50 & 1.49 & 1.42 & 1.47 & 1.46 \\
\hline HERA $F_{2}^{b}$ & {$[86,87]$} & 29 & 1.12 & 1.12 & 1.12 & 1.12 & 1.12 & 1.12 \\
\hline E886 & [88-90] & 104 & 1.27 & 1.25 & 1.29 & 1.28 & 1.30 & 1.31 \\
\hline E605 & {$[91]$} & 85 & 1.23 & 1.22 & 1.24 & 1.26 & 1.19 & 1.22 \\
\hline $\mathrm{CDF}$ & {$[92]$} & 29 & 1.47 & 1.44 & 1.52 & 1.42 & 1.44 & 1.48 \\
\hline D0 & {$[93-95]$} & 45 & 1.17 & 1.15 & 1.17 & 1.16 & 1.17 & 1.16 \\
\hline ATLAS high-mass DY $7 \mathrm{TeV}$ & {$[96]$} & 5 & 1.52 & 1.53 & 1.50 & 1.51 & 1.52 & 1.51 \\
\hline ATLAS low-mass DY $7 \mathrm{TeV}$ & {$[97]$} & 6 & 0.89 & 0.90 & 0.88 & 0.89 & 0.90 & 0.89 \\
\hline ATLAS $W, Z 7 \mathrm{TeV} 2010$ & {$[98]$} & 30 & 0.97 & 0.96 & 0.98 & 0.97 & 0.96 & 0.96 \\
\hline ATLAS $W, Z 7 \mathrm{TeV} 2011$ & {$[99]$} & 34 & 2.21 & 2.22 & 2.17 & 2.24 & 2.17 & 2.06 \\
\hline ATLAS $Z p_{T} 8 \mathrm{TeV}$ & {$[100]$} & 92 & 0.92 & 0.92 & 0.90 & 0.91 & 0.92 & 0.91 \\
\hline ATLAS jets $20117 \mathrm{TeV}$ & {$[101]$} & 31 & 1.11 & 1.13 & 1.11 & 1.11 & 1.12 & 1.12 \\
\hline ATLAS $t \bar{t}$ & {$[102-104]$} & 13 & 1.29 & 1.23 & 1.31 & 1.28 & 1.24 & 1.24 \\
\hline CMS $W$ asymmetry & {$[105,106]$} & 22 & 1.28 & 1.26 & 1.26 & 1.27 & 1.27 & 1.25 \\
\hline CMS Drell-Yan 2D 2011 & {$[107]$} & 110 & 1.27 & 1.26 & 1.26 & 1.26 & 1.26 & 1.26 \\
\hline CMS $W$ rapidity $8 \mathrm{TeV}$ & [108] & 22 & 1.03 & 1.01 & 1.00 & 0.98 & 1.03 & 0.99 \\
\hline $\mathrm{CMS} Z p_{T} 8 \mathrm{TeV}\left(p_{T}^{l l}, y_{l l}\right)$ & {$[109]$} & 28 & 1.31 & 1.30 & 1.34 & 1.32 & 1.31 & 1.31 \\
\hline CMS jets $7 \mathrm{TeV} 2011$ & {$[110]$} & 133 & 1.03 & 0.94 & 0.95 & 0.96 & 0.96 & 0.93 \\
\hline $\mathrm{CMS} t \bar{t}$ & {$[111-113]$} & 13 & 0.86 & 0.87 & 0.87 & 0.87 & 0.87 & 0.85 \\
\hline LHCb $Z 940 \mathrm{pb}$ & {$[114]$} & 9 & 1.43 & 1.45 & 1.47 & 1.41 & 1.46 & 1.45 \\
\hline $\mathrm{LHCb} Z \rightarrow e e 2 \mathrm{fb}$ & {$[115]$} & 17 & 1.14 & 1.12 & 1.14 & 1.12 & 1.12 & 1.13 \\
\hline $\mathrm{LHCb} W, Z \rightarrow \mu 7 \mathrm{TeV}$ & {$[116]$} & 29 & 1.93 & 1.83 & 1.88 & 1.84 & 1.84 & 1.86 \\
\hline $\mathrm{LHCb} W, Z \rightarrow \mu 8 \mathrm{TeV}$ & {$[117]$} & 30 & 1.64 & 1.56 & 1.54 & 1.58 & 1.55 & 1.52 \\
\hline $\operatorname{ATLAS} \sigma_{t} / \sigma_{\bar{t}} 7 \mathrm{TeV}$ & {$[12]$} & 1 & {$[0.32]$} & {$[0.39]$} & {$[0.37]$} & 0.33 & 0.34 & 0.30 \\
\hline $\operatorname{ATLAS} \sigma_{t} / \sigma_{\bar{t}} 8 \mathrm{TeV}$ & {$[13]$} & 1 & {$[3.54]$} & 3.13 & 3.15 & 3.34 & 3.25 & {$[3.72]$} \\
\hline $\operatorname{ATLAS} \sigma_{t} / \sigma_{\bar{t}} 13 \mathrm{TeV}$ & {$[14]$} & 1 & {$[0.04]$} & 0.04 & 0.05 & 0.04 & 0.04 & 0.04 \\
\hline $\mathrm{CMS} \sigma_{t} / \sigma_{\bar{t}} 8 \mathrm{TeV}$ & {$[16]$} & 1 & {$[0.10]$} & 0.13 & 0.13 & 0.11 & 0.12 & 0.09 \\
\hline $\mathrm{CMS} \sigma_{t} / \sigma_{\bar{t}} 13 \mathrm{TeV}$ & {$[17]$} & 1 & {$[0.31]$} & 0.31 & 0.33 & 0.31 & 0.30 & 0.30 \\
\hline $\mathrm{CMS} \sigma_{t+\bar{t}} 7 \mathrm{TeV}$ & {$[15]$} & 1 & {$[0.63]$} & 0.59 & 0.63 & 0.62 & 0.59 & 0.62 \\
\hline $\operatorname{ATLAS} d \sigma / d p_{T}(t) 7 \mathrm{TeV}$ & {$[12]$} & 5 & {$[2.87]$} & 2.87 & {$[2.87]$} & {$[2.87]$} & {$[2.88]$} & {$[2.87]$} \\
\hline $\operatorname{ATLAS} d \sigma / d p_{T}(\bar{t}) 7 \mathrm{TeV}$ & {$[12]$} & 5 & {$[12.0]$} & 12.0 & {$[12.1]$} & {$[12.0]$} & {$[12.0]$} & {$[11.9]$} \\
\hline ATLAS $d \sigma / d|y(t)| 7 \mathrm{TeV}$ & {$[12]$} & 4 & {$[1.31]$} & {$[1.32]$} & 1.31 & {$[1.30]$} & {$[1.31]$} & {$[1.31]$} \\
\hline ATLAS $d \sigma / d|y(\bar{t})| 7 \mathrm{TeV}$ & {$[12]$} & 4 & {$[0.07]$} & {$[0.07]$} & 0.06 & {$[0.07]$} & {$[0.07]$} & {$[0.07]$} \\
\hline $\operatorname{ATLAS}(1 / \sigma) d \sigma / d p_{T}(t) 7 \mathrm{TeV}$ & {$[12]$} & 4 & {$[2.79]$} & {$[2.79]$} & {$[2.79]$} & 2.79 & {$[2.79]$} & {$[2.79]$} \\
\hline $\operatorname{ATLAS}(1 / \sigma) d \sigma / d p_{T}(\bar{t}) 7 \mathrm{TeV}$ & {$[12]$} & 4 & {$[6.28]$} & {$[6.30]$} & {$[6.32]$} & 6.29 & {$[6.29]$} & {$[6.28]$} \\
\hline $\operatorname{ATLAS}(1 / \sigma) d \sigma / d|y(t)| 7 \mathrm{TeV}$ & {$[12]$} & 3 & {$[0.97]$} & {$[0.97]$} & {$[0.97]$} & {$[0.98]$} & 0.94 & 0.96 \\
\hline $\operatorname{ATLAS}(1 / \sigma) d \sigma / d|y(\bar{t})| 7 \mathrm{TeV}$ & {$[12]$} & 3 & {$[0.07]$} & {$[0.06]$} & {$[0.06]$} & {$[0.06]$} & 0.06 & 0.06 \\
\hline Total & & 4017 & 1.19 & 1.19 & 1.19 & 1.19 & 1.19 & 1.19 \\
\hline
\end{tabular}

Table 6. The $\chi^{2}$ for the NNLO PDF fits summarised in table 5, as well as for the PDF fit to the combination of data that we find to be optimal. Numbers in boldface denote the single top measurements included in each fit. Numbers in square brackets denote datasets not included in the fit, and are computed from each fit. 
of the specific single top measurement included in the fit. In each case, the $\chi^{2}$ fluctuates around its baseline value in a statistically insignificant way. Note that we consider a shift in the $\chi^{2}$ as statistically significant only if it is larger than one standard deviation of the $\chi^{2}$ distribution; that is the new $\chi^{2}$ lies outside the limits defined by $\frac{\chi^{2} N_{\text {dat }} \pm \sqrt{2 N_{\text {dat }}}}{N_{\text {dat }}}$, where $\chi^{2}$ is the value for the Baseline fit, and $N_{\text {dat }}$ is the number of data points which have been used to normalise the $\chi^{2}$. Overall, single top data are fairly well described, albeit with the following exceptions, already observed in section 3 .

First, the poor pre-fit $\chi^{2}$ observed in section 3 for the transverse momentum differential distributions does not improve at all when the data is included in a fit. By comparison with the rapidity distributions, which instead show an equally acceptable $\chi^{2}$ before and after the fit, we cannot envision any theoretical reason that could explain such a discrepancy. We therefore conclude that, unless this is understood, the differential distribution in the transverse momentum of the top antiquark should not be included in a PDF fit. Second, the comparatively large pre-fit $\chi^{2}$ for the ATLAS $8 \mathrm{TeV} \sigma_{t} / \sigma_{\bar{t}}$ measurement decreases each time single top data is included in the fit, although it reaches a not particularly good minimum of 3.13 in Fit 2. We therefore conclude that the ATLAS single top-quark to top-antiquark ratio at $8 \mathrm{TeV}$ should not be included in a fit.

Having established which datasets are not suitable for inclusion in a fit, we then assessed which are the most constraining on the PDFs among the remaining ones. A careful analysis of the results of all of the fits reported in table 5 led us to the conclusion that the ATLAS $7 \mathrm{TeV}$ normalised rapidity distributions are such datasets. We therefore included them in our Optimal fit, together with the ATLAS top-quark to top-antiquark ratios at 7 and $13 \mathrm{TeV}$, the CMS total cross section at $7 \mathrm{TeV}$, and the CMS top-quark to top-antiquark ratios at 8 and $13 \mathrm{TeV}$. Note that the Optimal fit is equivalent to Fit 5, except for the exclusion of the ATLAS top-quark to top-antiquark ratio at $8 \mathrm{TeV}$.

In figure 11 we compare the up, down and gluon PDFs for the Optimal fit to those for the Baseline fit (Fit 1). Specifically, we show the PDFs and their uncertainties (both $1 \sigma$ and $68 \%$ CL bands) normalised to the central value of the Baseline fit (left panel); and the relative uncertainties of the PDFs (right panel). Results are shown at $Q=m_{t}=$ 172.5 GeV. As can be seen from figure 11, the central values remain essentially stable, while PDF uncertainties are reduced. The largest reduction (by about one third of the baseline uncertainty) is observed for the up and down quark PDFs at $x \gtrsim 10^{-3}$. The regions of $x$ in which we see constraining power from the single top data is consistent with what we expected to see from our analysis of the observable-PDF correlations in section 4.1. For a discussion of the impact of single top data on the $u / d$ ratio, see section 4.5 .

Turning our attention to the other fits in our study, we find that the fit including absolute $p_{T}$ distributions is the least constraining (Fit 2), and that the fits including the normalised $p_{T}$ distributions (Fit 4) and the absolute rapidity distributions (Fit 3) are similarly constraining, with the former tending to be slightly more constraining. We attribute these results to two sources: firstly, we would expect that the fits including rapidity distributions are more constraining than those including transverse momentum distributions, because the former are more consistent with theoretical predictions than the latter are (see section 3); secondly, we would expect that the fits including normalised distributions 

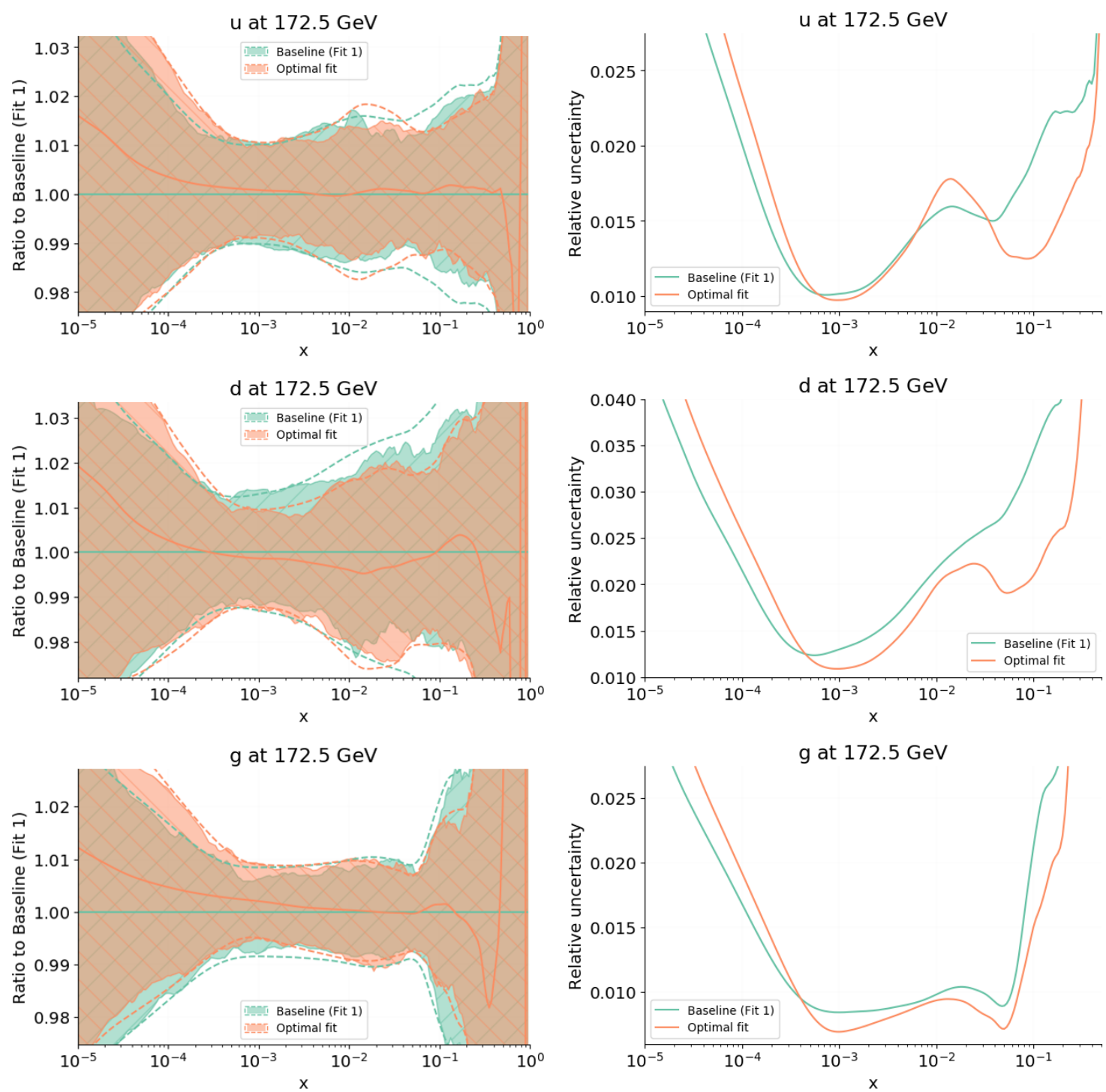

Figure 11. (Left) PDF plots of the up quark (top), down quark (middle) and gluon (bottom) for two global fits: Fit 1 (baseline) and the Optimal fit, as labelled in table 6. Each plot is normalised to the central value of Fit 1 , and is shown at $Q=m_{t}=172.5 \mathrm{GeV}$. The PDF uncertainty is shown as both a $68 \%$ CL hatched band and a $1 \sigma$ dashed line. (Right) The 1- $\sigma$ PDF uncertainties, normalised to their respective PDF central values.

are more constraining than those including absolute distributions, since the former benefit from uncertainty cancellations between the numerator and the denominator.

Having assessed the impact of the various datasets on PDFs, we can now revisit the question of whether the poor $\chi^{2}$ for the top-antiquark differential distributions and for the ATLAS top-quark to top-antiquark ratio at $8 \mathrm{TeV}$ could be driven by tensions with other measurement. To this purpose, we check whether a statistically significant shift in the $\chi^{2}$ of any of the baseline datasets has occurred when single top data has been fitted. We do this as defined at the start of section 4.3 and we find that no datasets exhibit such a shift. We 
do however see shifts of around $0.8 \sigma$ towards a lower $\chi^{2}$ for the CMS jets at $7 \mathrm{TeV}$ [110] in two cases: firstly, when fitting the absolute transverse momentum distributions (Fit 2), and secondly, when fitting the normalised rapidity distributions (Fit 5). Note that the shift is $0.7 \sigma$ for the Optimal fit. This suggests that 'CMS jets $7 \mathrm{TeV} 2011$ ' is highly correlated with some of the single top data. The next section will discuss such inter-dataset correlations in more detail.

We also repeat our fits by adding the single top data on top of a maximally consistent baseline dataset made up of HERA data only [84, 85]. Despite the more restrictive dataset, we run into the same inconsistencies reported above, namely we are not able to achieve an acceptable $\chi^{2}$ for the $7 \mathrm{TeV}$ top-antiquark transverse momentum differential distributions. This result demonstrates that the poor description of this dataset does not depend on tensions with other LHC measurements. We therefore conclude that the ATLAS differential distributions in the transverse momentum of the top antiquark are internally inconsistent (for further evidence obtained by analysing differential distributions at $8 \mathrm{TeV}$, see appendix A). Such an inconsistency should possibly require the experimental analysis to be reassessed in the future.

All of these findings allow us to summarise which combination of single top data is optimal for inclusion in the next generation of PDF fits: namely, the data used in the Optimal fit, that is the CMS total cross section at $7 \mathrm{TeV}$, the CMS top-quark to topantiquark ratios at 8 and $13 \mathrm{TeV}$, the ATLAS top-quark to top-antiquark ratios at 7 and $13 \mathrm{TeV}$, and the ATLAS normalised rapidity distributions for the top quark and the top antiquark at $7 \mathrm{TeV}$. This combination is best able to constrain the PDFs relevant to single top production, avoids data with unsatisfactorily poor theoretical descriptions, and minimises any possible residual theoretical ambiguities.

\subsection{Observable-observable correlations}

We now study the correlation between single top data and other datasets that are included in the Baseline fit. To calculate this correlation we replace $f$ with a second observable in eq. (4.1). In what follows we compute $\rho$ between single top data and two other classes of measurement: firstly, those which are largely gluon-initiated, and secondly, those which are EW-induced, and in particular are mediated by a $W$ boson. We choose the first class because single top production is sensitive to the gluon PDF, as was seen in section 4.1, and we choose the second class because single top production in the $t$-channel is mediated by a $W$ boson. We consistently compute observables at NNLO in pure QCD at a reference scale $Q=m_{t}=172.5 \mathrm{GeV}$, and use the NNPDF31_nnlo_as_0118 PDF set. We explicitly checked that correlation patterns are not affected if any of the fits presented in section 4 is used instead.

We start by looking at the jets data. Figure 12 shows the correlations between the ATLAS $7 \mathrm{TeV}$ top-quark $p_{T}$ distribution, with the single top data unnormalised (left panel) and normalised (right panel), and the CMS $7 \mathrm{TeV}$ jet data [110], for which we saw a $0.8 \sigma$ improvement in the $\chi^{2}$ upon fitting single top data. For the absolute single top distribution we see reasonably strong correlation for low values of the jet $p_{T}$, which turns into anticorrelation as the jet $p_{T}$ increases. Upon normalising the single top data, the correlations 

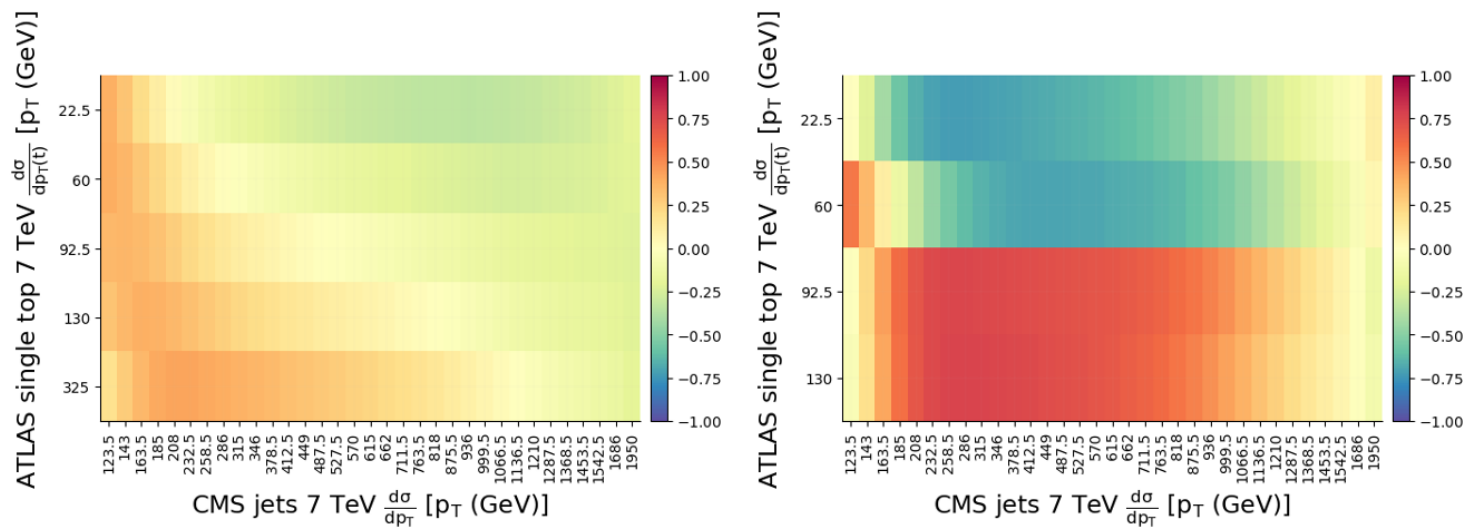

Figure 12. The correlations between bins of the CMS $7 \mathrm{TeV}$ inclusive jet dataset, which is binned in the $p_{T}$ of the jet, and both the ATLAS $7 \mathrm{TeV}$ single top-quark $p_{T}$ absolute (left panel) and normalised (right panel) distributions. The $\mathrm{CMS} 7 \mathrm{TeV}$ inclusive jet dataset is made up of five separate distributions, each of which represents a different rapidity bin. One bin is shown here, which is for $|y|<0.5$. These plots have been produced using theoretical predictions at NNLO in QCD with the NNPDF31_nnlo_as_0118 PDF set and $Q=m_{t}=172.5 \mathrm{GeV}$. The colour bar indicates the correspondence between $\rho$ and the colours in the plot.

become starker and a different correlation pattern emerges. The pattern becomes one where the lowest two rapidity bins of the single top data are generally strongly anti-correlated with the jet data, while the highest three rapidity bins are generally strongly correlated, with the correlation reaching $|\rho| \simeq 1$ in each case. Similar patterns of correlation are seen for the companion ATLAS $7 \mathrm{TeV}$ jet data [101], although no shift in the $\chi^{2}$ was seen for this dataset upon fitting single top data. Note that our fits in section 4.3 include five separate rapidity bins for the CMS jets data, the lowest of which we show here. A general trend of lower rapidities leading to stronger correlations is seen. Such a correlation has its origin in the gluon.

To study this further, we now look at another gluon-sensitive process: $t \bar{t}$ production. Figure 13 (left) shows the correlation between the ATLAS $7 \mathrm{TeV}$ top-quark $|y|$ distribution and the ATLAS $8 \mathrm{TeV} t \bar{t}$ rapidity distribution [104]. Here we see some correlation in some bins, most notably with the low rapidity bins of each distribution exhibiting strong correlation $(\rho \simeq 0.5)$ and the high rapidity bins of the $t \bar{t}$ data showing notable anticorrelation with the lowest three bins of the single top distribution $(\rho \simeq 0.3-0.5)$. However, the correlation with the $t \bar{t}$ data seems weaker than with the jets data. Clearly, while both single top production and $t \bar{t}$ are gluon-sensitive, $t \bar{t}$ tends to probe higher values of $x$ than both single top production and jets production. Hence, the inter-dataset correlation is stronger in the case of the latter datasets.

Next we look at correlations between single top data and other EW processes. Naively, we might expect that single top production data would exhibit non-negligible correlations with datasets such as weak boson production from LHCb [116] and ATLAS [99] at $7 \mathrm{TeV}$, because these datasets contain many data points for $W$-boson mediated processes, and therefore they may depend on similar contributions from the PDFs. However, for these datasets we do not see any notable correlations with the single top data. This is due to the 

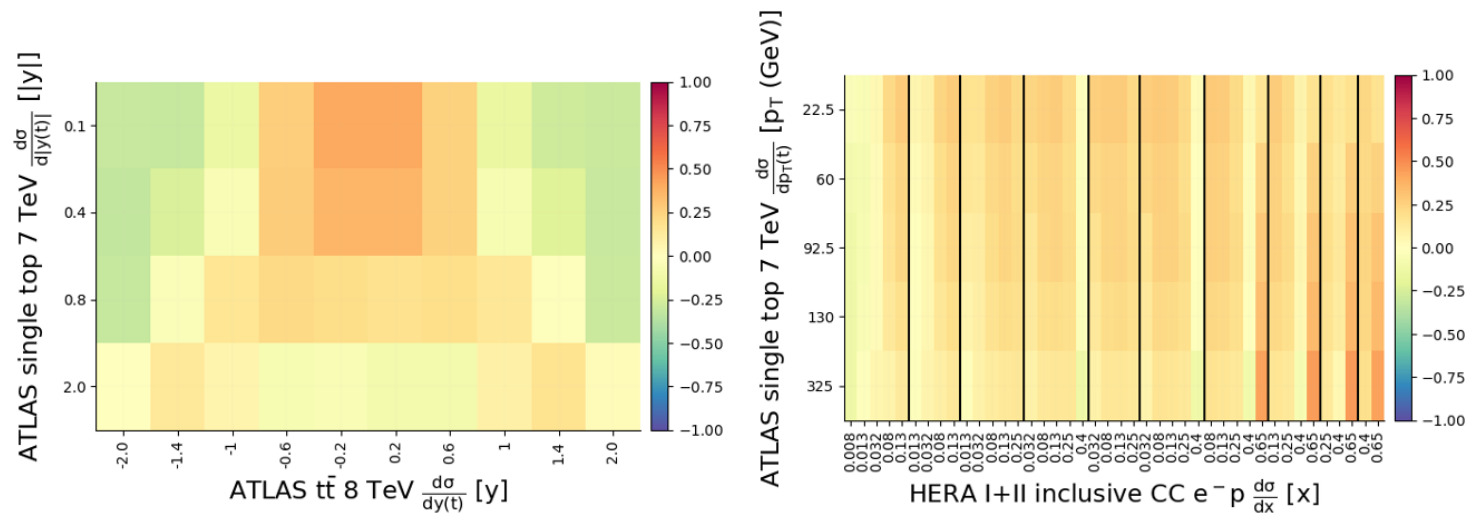

Figure 13. (Left) The correlations between bins of the ATLAS $8 \mathrm{TeV} t \bar{t}$ dataset, which is binned in the rapidity of the top quark, and the ATLAS $7 \mathrm{TeV}$ single top-quark absolute rapidity distribution. (Right) The correlations between the HERA combination $e^{-} p$ dataset and the absolute ATLAS $7 \mathrm{TeV}$ single top-quark transverse momentum distribution. The HERA data is binned in $Q^{2}$ and then within these bins, it is binned in Bjorken- $x$. The vertical black lines indicate the edges of the $Q^{2}$ bins. The lowest of these bins is for $Q^{2}=300 \mathrm{GeV}^{2}$ and the highest is for $Q^{2}=$ $30 \times 10^{3} \mathrm{GeV}^{2}$. Both plots have been produced using theoretical predictions at NNLO in QCD with the NNPDF31_nnlo_as_0118 PDF set and $Q=m_{t}=172.5 \mathrm{GeV}$. The colour bar indicates the correspondence between $\rho$ and the colours in the plot.

fact that single top production probes $u$ and $d$ in different regions of $x$ to either of these two datasets.

To investigate this further we consider another EW dataset, but this time from the HERA collider instead of the LHC. Here we look at HERA CC data from $e^{-} p$ collisions [84]. Figure 13 (right) shows the correlations between these data and the ATLAS $7 \mathrm{TeV}$ single top-quark $p_{T}$ distribution. Here we see regions of large correlation, up to $\rho \simeq 0.5-0.75$, contrary to what was seen for the LHC data we looked at previously. In particular, the correlation is largest for bins where $x=0.65$, with the correlation increasing with the single top-quark $p_{T}$. The correlation is relatively weak for the $x=0.4$ bins but is moderate for the $x \simeq 0.1$ and the $x=0.25$ bins. We therefore conclude that single top production data is indeed correlated with other EW observables, but that this effect is very dependent on the region of $x$ that the data probe. The HERA CC dataset covers similar regions of $x$ to the single top data, whereas the same is possibly not true for the aforementioned LHC data.

We conclude by noting that the picture we see here is similar to that which we saw in section 4.1. Namely, that single top production has a non-negligible dependence on the gluon PDF through its $b$-dependence, except when we consider measurements of $\sigma_{t} / \sigma_{\bar{t}}$ in which the gluon dependence largely cancels. This sensitivity, which we see via the correlation between single top data and both jets data and top-quark pair production data, supplements the up-quark and down-quark sensitivity that we expect. It also leads to notable correlations with HERA CC data, in which a similar $x$-region is probed.

This tells us that strong correlation is a necessary but not a sufficient condition for the data having an impact at the fit-level. For single top production we observe both 

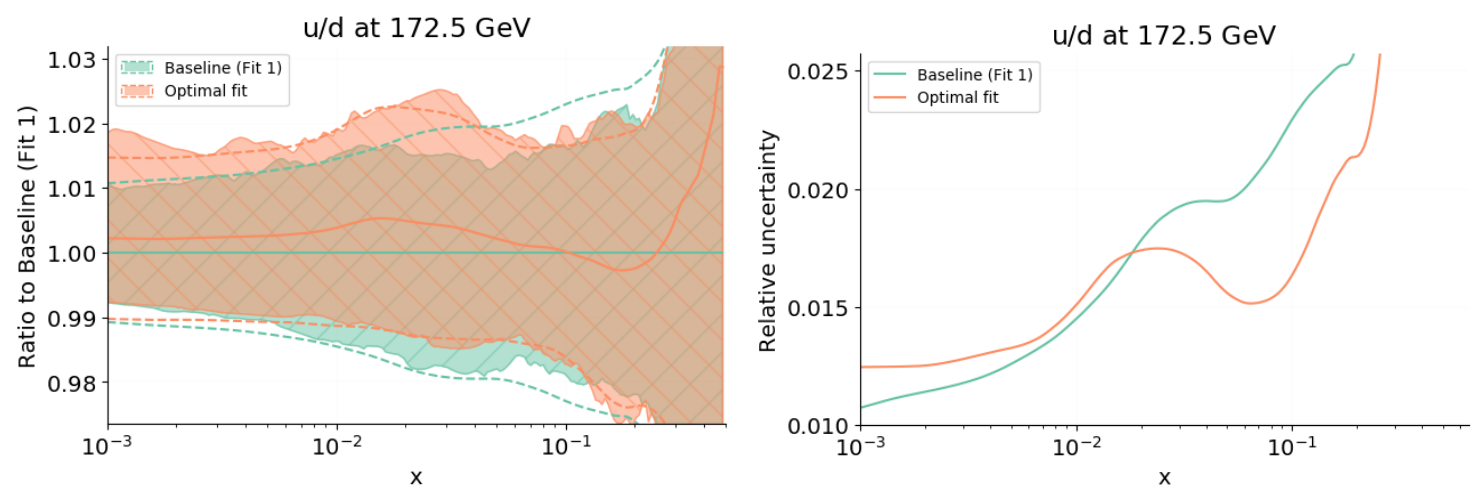

Figure 14. Same as figure 11 but for the up-quark to down-quark PDF ratio.

non-negligible PDF-observable correlations and notable correlations with HERA CC data, $t \bar{t}$ data and LHC jets data. However, the CMS jets data is the only data to show an improved data-theory agreement upon the inclusion of single top data in a fit, and this is true only in three of the five fits in which the single top data were included. Such a picture is expected to be modified as soon as more abundant and more precise single top data will become available.

\subsection{Phenomenological impact}

Here we discuss the ability of single top data to constrain quantities that are relevant for LHC phenomenology. We first consider the effect of single top data on the up-quark to down-quark PDF ratio, to which end we analyse the change in PDF uncertainties in predictions of the $W^{+} / W^{-}$ratio. We then turn our attention to the effect that single top data have on constraining parton luminosities.

Figure 14 shows how the $u / d$ ratio changes upon the inclusion of single top data in the PDF fit, where specifically we compare the Baseline fit with the Optimal fit, as defined in section 6 . We show how the PDF central value and its uncertainty changes upon including single top data (left-hand panel) and in addition how the relative uncertainty changes (right-hand panel). We find that this combination of data leads to a reduction in PDF uncertainties for the $u / d$ ratio, as we also saw for the PDF flavours discussed in section 4.3. In particular, a reduction in uncertainties is seen in the range $10^{-2} \lesssim x \lesssim 0.5$, which is largest (up to $25 \%$ ) at $x \simeq 0.003$. This corroborates the discussion in section 4.1 in which we said that single top data, and in particular measurements of $\sigma_{t} / \sigma_{\bar{t}}$, are indeed sensitive to $u / d$, and that this is true for a slightly larger range than was found in ref. [12].

The $u / d$ ratio is an important quantity for predictions of observables such as $W$ and $Z$ production, which have been measured very precisely at the $13 \mathrm{TeV}$ LHC run by ATLAS [122]. In particular the measurement of the $W^{+} / W^{-}$inclusive cross section ratio is very sensitive to the $u / d$ ratio. To quantify the difference that including single top data in PDF fits makes on such predictions, we compute the $W^{+} / W^{-}$ratio at NNLO at $\sqrt{s}=13 \mathrm{TeV}$ using the Baseline and the Optimal fit including the constraints provided by single top data and we compare them to the ATLAS experimental result. Results are 


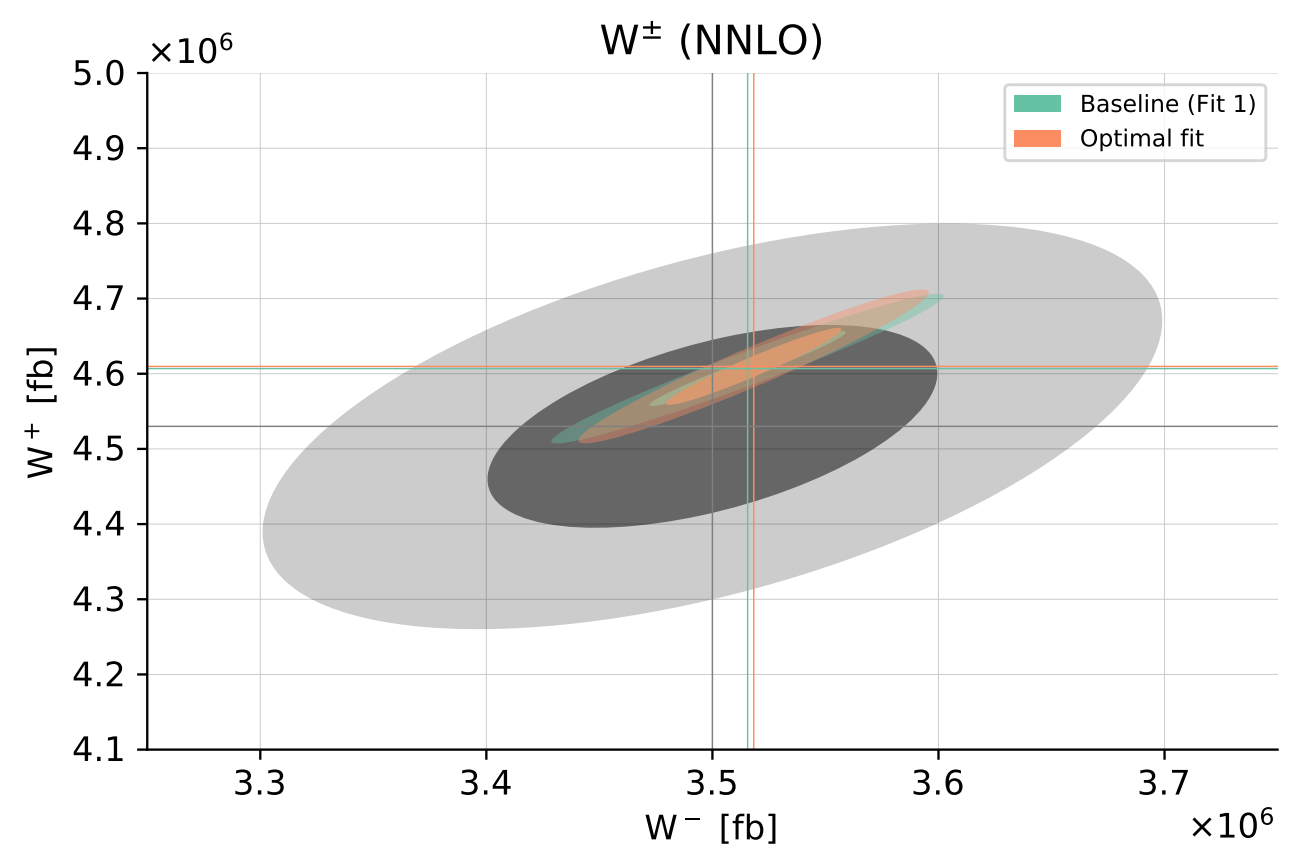

Figure 15. Theoretical predictions for the $W^{+}$and $W^{-}$cross section at NNLO obtained with the Baseline fit (green) and the Optimal fit (orange), compared to the $13 \mathrm{TeV}$ measurement performed by ATLAS [122]. The 68\% CL and $90 \%$ CL ellipses for the theoretical predictions are represented in dark and light shades, respectively. Correspondingly, the same CLs are plotted for the experimental measurement in dark and light grey.

displayed in figure 15. We see that the central value is slightly shifted by the inclusion of the single top data, but it is still well within the experimental $1 \sigma$ ellipse. The PDF uncertainty on the other hand remains almost unchanged. Still it is significantly smaller than the data uncertainty: a consistency test could be made more stringent only by increasing the precision of the experimental measurement.

We now broaden the scope of our discussion, by looking at the effect of single top data on parton flavours more generally. Specifically, we study how the relative uncertainties on a range of parton luminosities change upon including single top data in a PDF fit. We do this because parton luminosities, which describe the partonic content of two colliding protons, are in fact the relevant entities for the LHC. Note that we adopt the definition of the parton luminosity given in ref. [123].

Figure 16 compares the relative uncertainty on the gluon-gluon, gluon-quark, quarkquark, and quark-antiquark parton luminosities at $\sqrt{s}=13 \mathrm{TeV}$ (whereby a sum over all quark or antiquark flavours is performed), and figure 17 compares the relative uncertainty on the $u \bar{d}$ luminosity, to which single top data are expected to be particularly sensitive. The uncertainty is plotted in the invariant mass $M_{X}$ and the rapidity $y$ of the final state. The plots on the left-hand side show the relative uncertainties on the parton luminosities for the Optimal fit. The results on the right-hand side show the relative difference in uncertainties between the Baseline Fit (Fit 1) and the Optimal fit. The relative difference is found by taking the uncertainty for the Baseline fit away from the uncertainty for the Optimal 

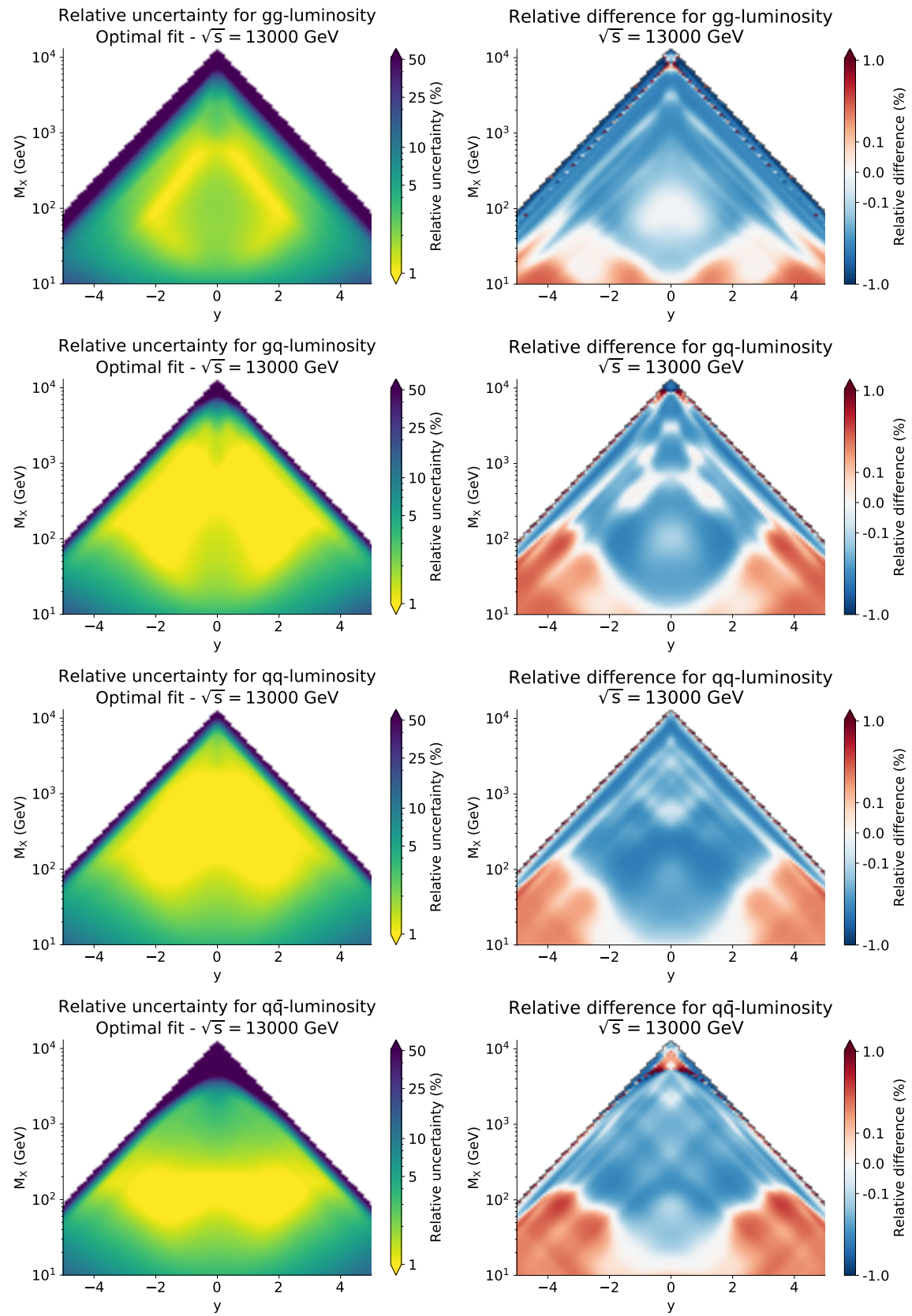

Figure 16. The parton luminosities as a function of the invariant mass $M_{X}$ and the rapidity $y$ of the final state at $\sqrt{s}=13 \mathrm{TeV}$. From top to bottom, the results shown are for the gluon-gluon, gluon-quark, quark-quark, and quark-antiquark luminosities. The results on the left-hand side show the relative uncertainties on the parton luminosities for the Optimal fit. For these plots the colour bars indicate the relative uncertainty, with yellow representing uncertainties of around $1 \%$. The results on the right-hand side show the relative difference in uncertainties between the Baseline Fit (Fit 1) and the Optimal fit. The relative difference is found by taking the uncertainty for the Baseline fit away from the uncertainty for the Optimal fit, and normalising to the former. Therefore, relative decreases in the uncertainty are shown in blue and relative increases are shown in red. 

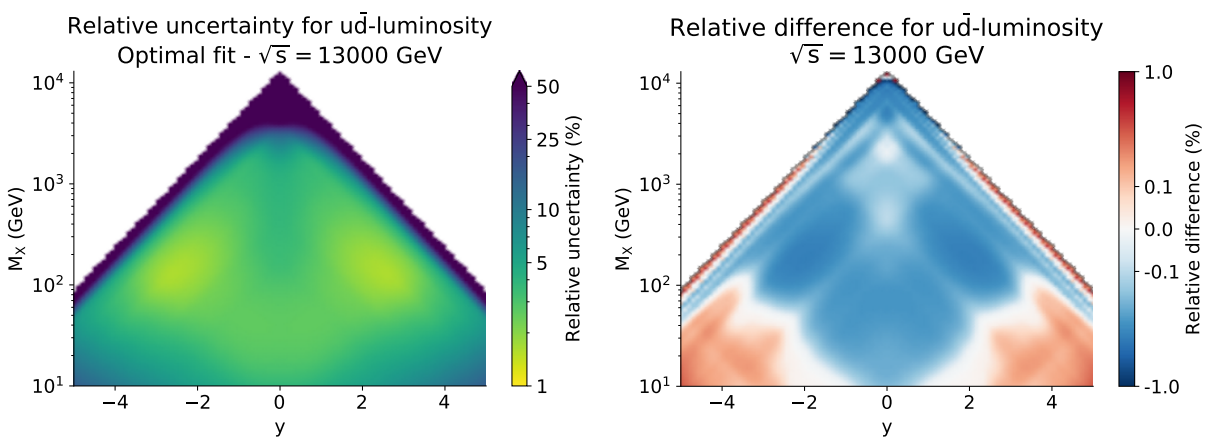

Figure 17. Same as figure 16 but for the $u \bar{d}$ luminosity.

fit, and normalising to the former. We see that for each combination of partons, there is a reduction in uncertainties for regions where the luminosities are already reasonably well constrained. This is most obvious for the luminosities involving quarks, which is as expected given that we saw a more significant decrease in PDF uncertainties for the up quark and the down quark compared to the gluon (see figure 11).

\section{Conclusions}

In this paper we systematically studied the impact of $t$-channel single top-quark and topantiquark production data from the LHC on the determination of the proton PDFs. Specifically, we considered total cross sections, top-antitop cross section ratios, and differential distributions measured by the ATLAS and CMS experiments at centre-of-mass energies of 7, 8 and $13 \mathrm{TeV}$. We presented a critical appraisal of the data, studying in particular how their description is affected by the theoretical details that enter the computation of the corresponding observables: QCD corrections, here systematically extended up to NNLO for the first time; EW corrections, possibly mixed with QCD corrections, up to NLO; the flavour scheme, either $5 \mathrm{FS}$ or $4 \mathrm{FS}$; and the value of the bottom-quark matching point. We performed a series of fits to the data within the NNPDF3.1 framework at NNLO, demonstrated the constraining power of the data, and their correlation with the rest of the dataset.

We found that the data is overall fairly well described with NNLO QCD in the 5FS. The effect of NNLO QCD corrections is typically of the order of few percent on the total cross sections and on the distributions differential in the rapidity of the top quark and top antiquark; they can be larger (up to 10\%) for the distributions differential in the transverse momentum of the top quark and top antiquark, especially for the highest $p_{T}$ bins. In comparison, NLO EW and mixed $\mathrm{EW} \times \mathrm{QCD}$ corrections account for a sub-percent effect, which is currently negligible in comparison to the data and the PDF uncertainty. We also found that the $4 \mathrm{FS}$, which might in principle be more adequate than the 5FS to describe single top data as it takes into account bottom-quark mass corrections, typically leads to lower predictions that are in worse agreement with the data, unless one chooses a smaller factorisation scale. Furthermore, single top total cross sections at NLO decrease 
by about $5-7 \%(10-12 \%)$ if the bottom-quark matching point $\mu_{b}$ in the $5 \mathrm{FS}$ is raised to twice (five times) the mass of the bottom quark, in such a way that they become closer to the $4 \mathrm{FS}$ results. The effect would be significantly smaller if the 5FS cross section had been computed at NNLO, as shown in ref. [31]. Further studies aiming to determine the value of $\mu_{b}$ that minimises the effect of $m_{b}$ power corrections in a 5FS, or aiming to compute the single top distributions in a matched scheme in order to explicitly include such $m_{b}$ power corrections in the theoretical predictions, will become necessary to describe single top data within a percent accuracy, once their experimental precision is improved.

Despite our careful investigation of all of the theoretical assumptions detailed above, we observed two significant inconsistencies between data and theory, for which we are unable to envision a satisfactory explanation. The first concerns the ratio of single topquark to top-antiquark total cross section from ATLAS at $8 \mathrm{TeV}$; the second concerns the distributions (both absolute and normalised) differential in the top-antiquark transverse momentum. In the first case, we noted that the analogous measurement from CMS, which differs from the ATLAS one by approximately $\sqrt{2} \sigma$, is instead very well described; in the latter case, we noted that the analogous measurement from ATLAS at $8 \mathrm{TeV}$ (albeit experimental correlations are not available) is also very well described.

We then assessed the sensitivity of PDFs to the single top data under scrutiny by performing a series of fits whereby we supplemented the NNPDF3.1 dataset (from which we removed jet data that lacks NNLO QCD $C$-factors) with suitable combinations of single top data. We found that the data-theory inconsistencies observed before the fit are not resolved. Because we did not observe any apparent tension with the rest of the dataset, even when this was reduced to a maximally consistent dataset made up entirely of HERA data, we concluded that transverse momentum differential distributions should not be included in a fit. In light of these results, the optimal combination of single top data that we recommend to include in a fit is made of the following measurements: the available ratios of single topquark to top-antiquark measurements (from ATLAS at 7, 8 and $13 \mathrm{TeV}$ and from CMS at 8 and $13 \mathrm{TeV}$ ), the total cross section if the former is not available (for CMS at $7 \mathrm{TeV}$ ), and the top-quark and top-antiquark rapidity distributions (for ATLAS at $7 \mathrm{TeV}$ ). Concerning the latter, we prefer the normalised distribution. While this choice neglects unavailable experimental correlations with the total cross sections, it minimises unaccounted theoretical uncertainties (such as missing higher order QCD and EW uncertainties, the dependence on the flavour scheme or on the bottom matching threshold).

Finally, we demonstrated that this optimal combination of data effectively constrains the light quark and the gluon PDFs by reducing their relative uncertainty by a fraction of a percent in the region $10^{-3} \leq x \leq 0.5$ (with a reduction of up to $25 \%$ on the ratio $u / d$ around $x \sim 0.1$ ). As expected, for the $u$ and $d$ such an effect is driven by the EW nature of the process, while for the gluon it is driven by the perturbative generation of the initiating $b$-quark. The information provided by single top data is complementary to that provided by other processes in the NNPDF3.1 dataset, both EW-induced, such as $W$-boson production or CC DIS, and gluon-sensitive, such as jet or top-pair production. Single top production will therefore be a useful ingredient in the next generation of PDF determinations. 


\begin{tabular}{|ccccc|}
\hline Distribution & Kinematic range & $N_{\text {dat }}$ & $\chi_{\mathrm{NLO}}^{2(5 \mathrm{FS})}$ & $\chi_{\mathrm{NNLO}}^{2(5 \mathrm{FS})}$ \\
\hline$d \sigma / d p_{T}(t)$ & $0<p_{T}(t)<300 \mathrm{GeV}$ & 5 & 0.71 & 0.46 \\
$d \sigma / d p_{T}(\bar{t})$ & $0<p_{T}(\bar{t})<300 \mathrm{GeV}$ & 4 & 0.90 & 1.36 \\
$d \sigma / d|y(t)|$ & $0<|y(t)|<2.2$ & 4 & 0.17 & 0.29 \\
$d \sigma / d|y(\bar{t})|$ & $0<|y(\bar{t})|<2.2$ & 4 & 0.40 & 0.37 \\
$(1 / \sigma) d \sigma / d p_{T}(t)$ & $0<p_{T}(t)<300 \mathrm{GeV}$ & 4 & 0.80 & 0.05 \\
$(1 / \sigma) d \sigma / d p_{T}(\bar{t})$ & $0<p_{T}(\bar{t})<300 \mathrm{GeV}$ & 3 & 0.02 & 0.51 \\
$(1 / \sigma) d \sigma / d|y(t)|$ & $0<|y(t)|<2.2$ & 3 & 0.24 & 0.19 \\
$(1 / \sigma) d \sigma / d|y(\bar{t})|$ & $0<|y(\bar{t})|<2.2$ & 3 & 0.33 & 0.34 \\
\hline
\end{tabular}

Table 7. The values of the $\chi^{2}$, eq. (3.2), computed for all of the datasets presented in ref. [13]. We indicate the distribution, its kinematic range, and the corresponding number of data points, $N_{\text {dat }}$. The bin edges for the transverse momentum distributions are 0, 50, 100, 150, 200 and $300 \mathrm{GeV}$, while for the rapidity distributions they are $0,0.3,0.7,1.3$ and 2.2. Theoretical predictions are computed at either NLO or NNLO accuracy in the 5FS in pure QCD; the input PDFs are taken from the NNPDF31_nlo_as_0118 and NNPDF31_nnlo_as_0118 sets, respectively. Experimental correlations are not provided, therefore they are not included in the $\chi^{2}$ computation.

\section{A The ATLAS $8 \mathrm{TeV}$ differential distributions}

In this appendix we illustrate the ATLAS data of ref. [13], that is, single top-quark and top-antiquark differential distributions measured at a centre-of-mass energy of $8 \mathrm{TeV}$. As discussed in section 2, these are not included in the fits presented in this work because no information on how experimental uncertainties are correlated is provided. Nevertheless, we believe that a data-theory comparison, along the lines of what was presented in section 3 , is instructive, in particular to investigate whether the discrepancies observed in the ATLAS $7 \mathrm{TeV}$ data persist or not.

The data is compared to the corresponding QCD (at $\mathrm{NLO}$ and $\mathrm{NNLO}$ ), and $\mathrm{QCD} \times \mathrm{EW}$ (at NLO) theoretical predictions for both absolute and normalised differential cross sections of top-quark production in figure 18 and of top-antiquark production in figure 19 . Theoretical predictions are computed with the same computational settings, physical parameters and input PDFs as delineated in sections 3.1-3.2. The error bands correspond to the $68 \%$ CL PDF uncertainty. The features of the data are summarised in table 7 , where we display, for each distribution, its kinematic range, the number of data points, $N_{\text {dat }}$, and the values of the $\chi^{2}$ obtained at NLO and NNLO in the 5FS (pure QCD). As in section 3, the last bin of the normalised distributions is removed from the computation of the $\chi^{2}$, but not from the data-theory comparisons displayed in figures 18-19.

As is apparent from table 7 and figures 18-19, the description of the data provided by NNPDF3.1 is qualitatively and quantitatively excellent for all the differential distributions. The impact of EW corrections is negligible, while the impact of NNLO QCD corrections is moderate. These lead to a general improvement of the $\chi^{2}$, except for the distributions differential in the transverse momentum of the top antiquark, and in the absolute rapidity of the top quark. This seems to be a consequence of the fact that the NNLO prediction for both the largest $p_{T}(\bar{t})$ and $|y(t)|$ bins is farther from the data than its NLO counterpart. All 

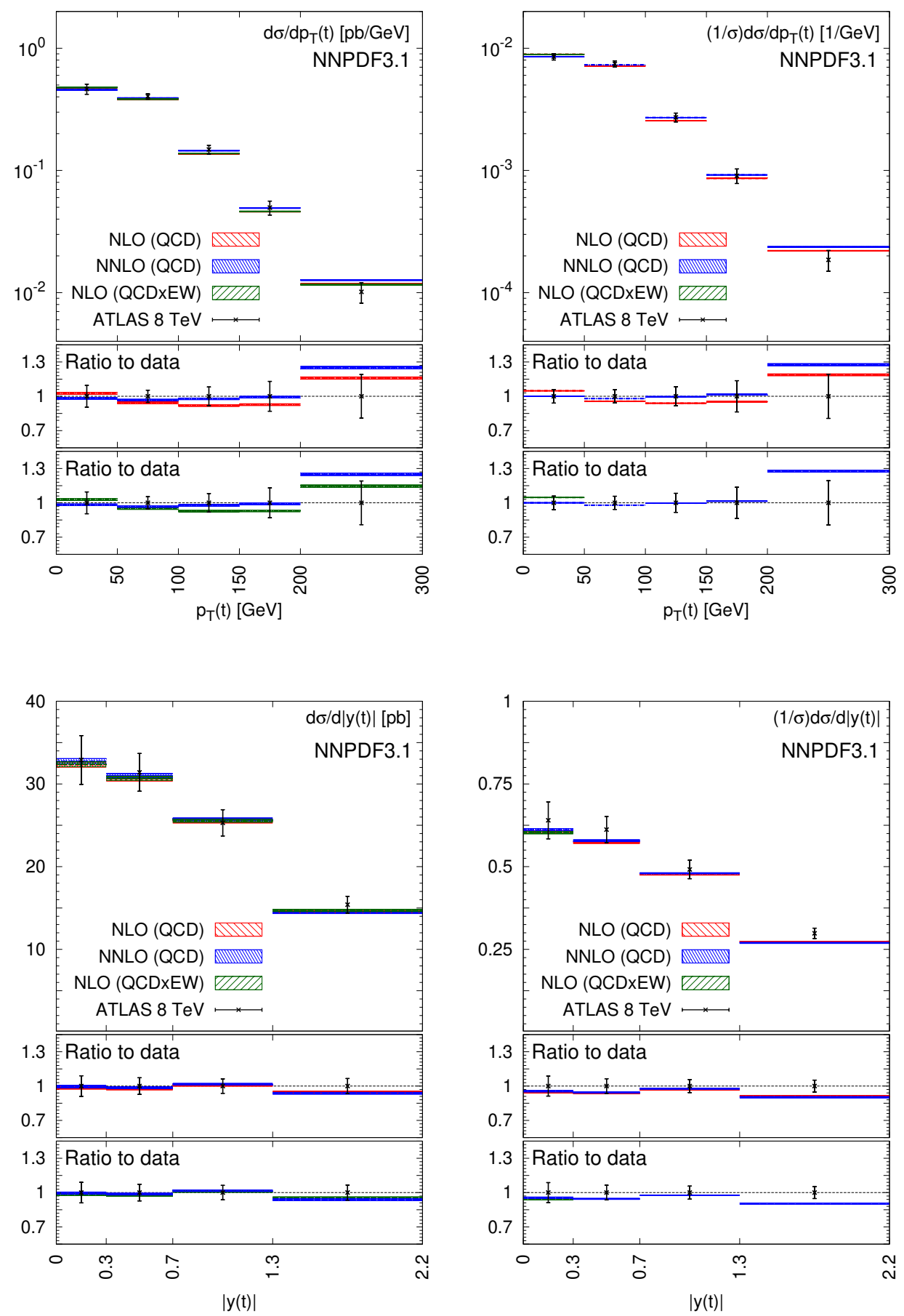

Figure 18. Same as figure 3, but for the ATLAS data of ref. [13]. 

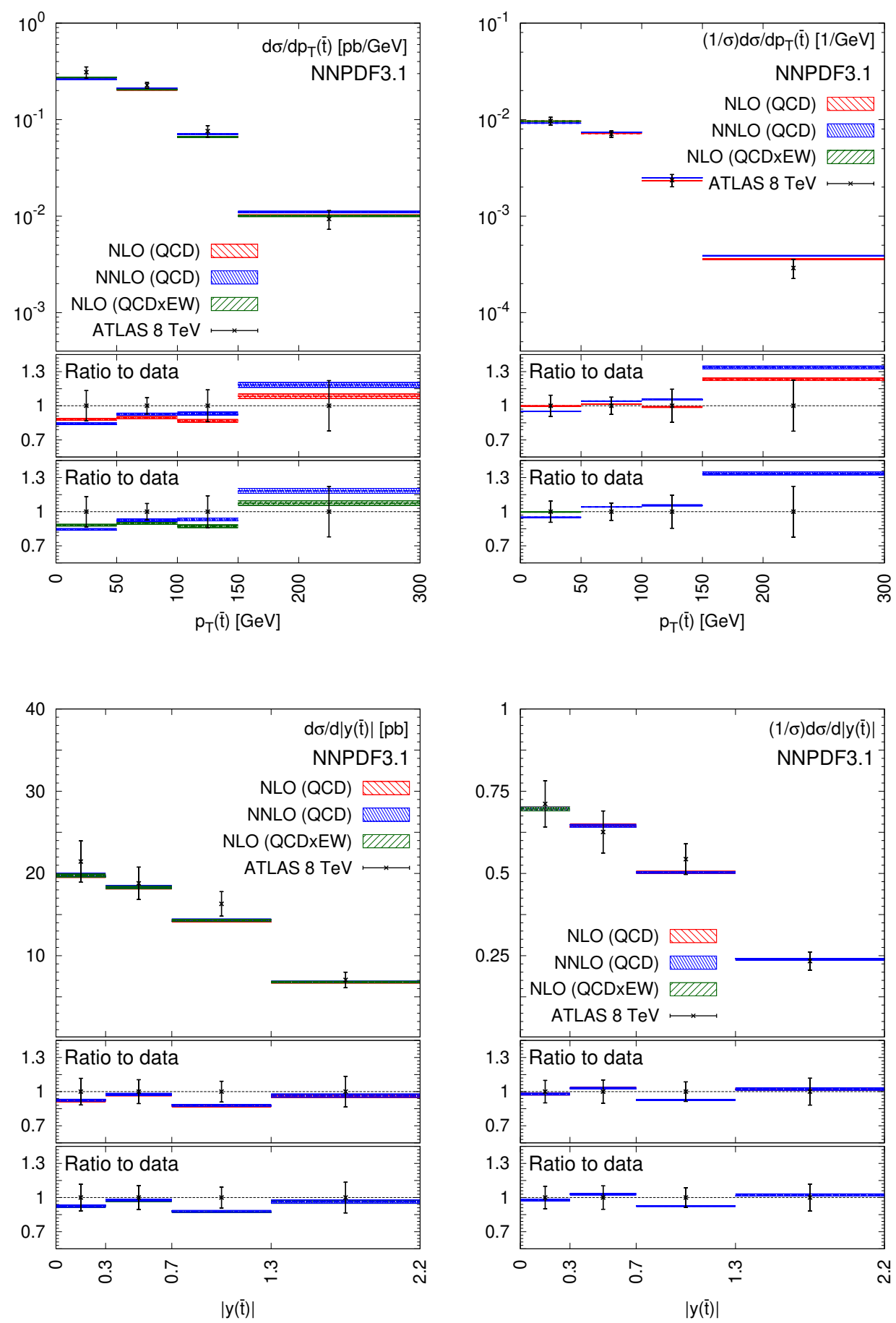

Figure 19. Same as figure 4, but for the ATLAS data of ref. [13]. 
of the $\chi^{2}$, however, remain perfectly acceptable; any further conclusion must be reassessed when experimental correlations become available.

The overall excellent description of the data includes, most notably, the distributions differential in the transverse momentum of the top antiquark, for which we found large datatheory tensions in the analogous ATLAS measurement at a centre-of-mass energy of $7 \mathrm{TeV}$ (see section 3). We interpret this result as further evidence of the fact that the discrepancy observed in section 3 is likely not to have a theoretical origin. We believe that this specific measurement deserves further investigation, possibly on the original experimental analysis, and should therefore not be included in a PDF fit.

\section{Acknowledgments}

The Authors would like to thank Jun Gao for providing them with the NNLO computation of refs. [25, 26], Marco Zaro for support with MADGRAPH5_AMC@NLO, Zahari Kassabov for his help with the REPORTENGINE software, Lucian Harland-Lang for a critical reading of the manuscript, Reinhard Schwienhorst and Alberto Orso Maria Iorio for clarifications on the ATLAS and CMS experimental data, respectively, and the members of the NNPDF Collaboration, in particular Stefano Forte, Zahari Kassabov, Juan Rojo and Luca Rottoli, for useful comments on the manuscript. E.R.N. is supported by the European Commission through the Marie Skłodowska-Curie Action ParDHonS_FFs.TMDs (grant number 752748). M.U. is funded by the Royal Society grant DH/150088 and supported by the STFC consolidated grant ST/L000681/1 and the Royal Society grant RGF/EA/180148. C.V. is supported by the STFC grant ST/R504671/1.

Open Access. This article is distributed under the terms of the Creative Commons Attribution License (CC-BY 4.0), which permits any use, distribution and reproduction in any medium, provided the original author(s) and source are credited.

\section{References}

[1] J. Alwall et al., Is $V\left({ }_{t b}\right) \simeq 1$ ?, Eur. Phys. J. C 49 (2007) 791 [hep-ph/0607115] [inSPIRE].

[2] Q.-H. Cao and B. Yan, Determining $V_{t b}$ at electron-positron colliders, Phys. Rev. D 92 (2015) 094018 [arXiv: 1507.06204] [inSPIRE].

[3] CMS collaboration, Measurement of the top quark mass using single top quark events in proton-proton collisions at $\sqrt{s}=8 \mathrm{TeV}$, Eur. Phys. J. C 77 (2017) 354 [arXiv: 1703.02530] [INSPIRE].

[4] S. Alekhin, S. Moch and S. Thier, Determination of the top-quark mass from hadro-production of single top-quarks, Phys. Lett. B 763 (2016) 341 [arXiv:1608.05212] [INSPIRE].

[5] T.M.P. Tait and C.P. Yuan, Single top quark production as a window to physics beyond the standard model, Phys. Rev. D 63 (2000) 014018 [hep-ph/0007298] [INSPIRE].

[6] J.A. Aguilar-Saavedra, Single top quark production at LHC with anomalous Wtb couplings, Nucl. Phys. B 804 (2008) 160 [arXiv:0803.3810] [INSPIRE]. 
[7] N.P. Hartland et al., A Monte Carlo global analysis of the Standard Model Effective Field Theory: the top quark sector, JHEP 04 (2019) 100 [arXiv:1901.05965] [INSPIRE].

[8] J. Gao, C.S. Li, L.L. Yang and H. Zhang, Search for anomalous top quark production at the early LHC, Phys. Rev. Lett. 107 (2011) 092002 [arXiv:1104.4945] [InSPIRE].

[9] Q.-H. Cao, B. Yan, J.-H. Yu and C. Zhang, A General Analysis of Wtb anomalous Couplings, Chin. Phys. C 41 (2017) 063101 [arXiv:1504.03785] [INSPIRE].

[10] S. Alekhin, J. Blümlein, S. Moch and R. Plačakyte, Isospin asymmetry of quark distributions and implications for single top-quark production at the LHC, Phys. Rev. D 94 (2016) 114038 [arXiv: 1508.07923] [inSPIRE].

[11] S. Alekhin, J. Blümlein, S. Moch and R. Plačakyte, Parton distribution functions, $\alpha_{s}$ and heavy-quark masses for LHC Run II, Phys. Rev. D 96 (2017) 014011 [arXiv:1701.05838] [INSPIRE].

[12] ATLAS collaboration, Comprehensive measurements of $t$-channel single top-quark production cross sections at $\sqrt{s}=7 \mathrm{TeV}$ with the ATLAS detector, Phys. Rev. D 90 (2014) 112006 [arXiv: 1406.7844] [INSPIRE].

[13] ATLAS collaboration, Fiducial, total and differential cross-section measurements of $t$-channel single top-quark production in pp collisions at $8 \mathrm{TeV}$ using data collected by the ATLAS detector, Eur. Phys. J. C 77 (2017) 531 [arXiv:1702.02859] [InSPIRE].

[14] ATLAS collaboration, Measurement of the inclusive cross-sections of single top-quark and top-antiquark $t$-channel production in pp collisions at $\sqrt{s}=13 \mathrm{TeV}$ with the ATLAS detector, JHEP 04 (2017) 086 [arXiv: 1609.03920] [INSPIRE].

[15] CMS collaboration, Measurement of the Single-Top-Quark t-Channel Cross Section in pp Collisions at $\sqrt{s}=7 \mathrm{TeV}$, JHEP 12 (2012) 035 [arXiv:1209.4533] [INSPIRE].

[16] CMS collaboration, Measurement of the t-channel single-top-quark production cross section and of the $\left|V_{t b}\right|$ CKM matrix element in pp collisions at $\sqrt{s}=8 \mathrm{TeV}$, JHEP 06 (2014) 090 [arXiv: 1403.7366] [INSPIRE].

[17] CMS collaboration, Cross section measurement of $t$-channel single top quark production in pp collisions at $\sqrt{s}=13 \mathrm{TeV}$, Phys. Lett. B 772 (2017) 752 [arXiv:1610.00678] [InSPIRE].

[18] G. Bordes and B. van Eijk, Calculating QCD corrections to single top production in hadronic interactions, Nucl. Phys. B 435 (1995) 23 [INSPIRE].

[19] R. Pittau, Final state QCD corrections to off-shell single top production in hadron collisions, Phys. Lett. B 386 (1996) 397 [hep-ph/9603265] [INSPIRE].

[20] T. Stelzer, Z. Sullivan and S. Willenbrock, Single top quark production via $W$-gluon fusion at next-to-leading order, Phys. Rev. D 56 (1997) 5919 [hep-ph/9705398] [INSPIRE].

[21] B.W. Harris, E. Laenen, L. Phaf, Z. Sullivan and S. Weinzierl, The Fully Differential Single Top Quark Cross-Section in Next to Leading Order QCD, Phys. Rev. D 66 (2002) 054024 [hep-ph/0207055] [INSPIRE].

[22] P. Falgari, P. Mellor and A. Signer, Production-decay interferences at NLO in QCD for t-channel single-top production, Phys. Rev. D 82 (2010) 054028 [arXiv:1007.0893] [INSPIRE].

[23] R. Schwienhorst, C.P. Yuan, C. Mueller and Q.-H. Cao, Single top quark production and decay in the t-channel at next-to-leading order at the LHC, Phys. Rev. D 83 (2011) 034019 [arXiv: 1012.5132] [INSPIRE]. 
[24] M. Brucherseifer, F. Caola and K. Melnikov, On the NNLO QCD corrections to single-top production at the LHC, Phys. Lett. B 736 (2014) 58 [arXiv:1404.7116] [INSPIRE].

[25] E.L. Berger, J. Gao, C.P. Yuan and H.X. Zhu, NNLO QCD Corrections to t-channel Single Top-Quark Production and Decay, Phys. Rev. D 94 (2016) 071501 [arXiv:1606.08463] [INSPIRE].

[26] E.L. Berger, J. Gao and H.X. Zhu, Differential Distributions for t-channel Single Top-Quark Production and Decay at Next-to-Next-to-Leading Order in QCD, JHEP 11 (2017) 158 [arXiv: 1708.09405] [INSPIRE].

[27] M. Beccaria, G. Macorini, F.M. Renard and C. Verzegnassi, Single top production in the t-channel at LHC: A realistic test of electroweak models, Phys. Rev. D 74 (2006) 013008 [hep-ph/0605108] [INSPIRE].

[28] E. Mirabella, Electroweak Corrections to t-channel single top production at the LHC, Nuovo Cim. B 123 (2008) 1111 [arXiv:0811.2051] [InSPIRE].

[29] D. Bardin, S. Bondarenko, L. Kalinovskaya, V. Kolesnikov and W. von Schlippe, Electroweak Radiative Corrections to Single-top Production, Eur. Phys. J. C 71 (2011) 1533 [arXiv: 1008.1859] [INSPIRE].

[30] J.M. Campbell, R. Frederix, F. Maltoni and F. Tramontano, Next-to-Leading-Order Predictions for t-Channel Single-Top Production at Hadron Colliders, Phys. Rev. Lett. 102 (2009) 182003 [arXiv:0903.0005] [INSPIRE].

[31] V. Bertone, A. Glazov, A. Mitov, A. Papanastasiou and M. Ubiali, Heavy-flavor parton distributions without heavy-flavor matching prescriptions, JHEP 04 (2018) 046 [arXiv: 1711.03355] [INSPIRE].

[32] NNPDF collaboration, Parton distributions from high-precision collider data, Eur. Phys. J. C 77 (2017) 663 [arXiv: 1706.00428] [INSPIRE].

[33] D0 collaboration, Measurement of the t-channel single top quark production cross section, Phys. Lett. B 682 (2010) 363 [arXiv:0907.4259] [INSPIRE].

[34] CDF collaboration, Observation of Single Top Quark Production and Measurement of $\left|V_{t b}\right|$ with CDF, Phys. Rev. D 82 (2010) 112005 [arXiv: 1004.1181] [INSPIRE].

[35] ATLAS collaboration, Measurement of the t-channel single top-quark production cross section in pp collisions at $\sqrt{s}=7 \mathrm{TeV}$ with the ATLAS detector, Phys. Lett. B 717 (2012) 330 [arXiv:1205.3130] [INSPIRE].

[36] CMS collaboration, Measurement of the t-channel single top quark production cross section in pp collisions at $\sqrt{s}=7 \mathrm{TeV}$, Phys. Rev. Lett. 107 (2011) 091802 [arXiv:1106.3052] [INSPIRE].

[37] NNPDF collaboration, A first determination of parton distributions with theoretical uncertainties, Eur. Phys. J. C (2019) 79:838 [arXiv:1905.04311] [InSPIRE].

[38] NNPDF collaboration, Parton Distributions with Theory Uncertainties: General Formalism and First Phenomenological Studies, Eur. Phys. J. C 79 (2019) 931 [arXiv: 1906.10698] [INSPIRE].

[39] CMS collaboration, Measurement of Top Quark Polarisation in T-Channel Single Top Quark Production, JHEP 04 (2016) 073 [arXiv:1511.02138] [INSPIRE]. 
[40] CMS collaboration, Measurement of differential cross sections and charge ratios for t-channel single top quark production in proton-proton collisions at $\sqrt{s}=13 \mathrm{TeV}$, Eur. Phys. J. C 80 (2020) 370 [arXiv:1907.08330] [INSPIRE].

[41] M. Brucherseifer, F. Caola and K. Melnikov, $\mathcal{O}\left(\alpha_{s}^{2}\right)$ corrections to fully-differential top quark decays, JHEP 04 (2013) 059 [arXiv:1301.7133] [INSPIRE].

[42] J. Gao and A.S. Papanastasiou, Top-quark pair-production and decay at high precision, Phys. Rev. D 96 (2017) 051501 [arXiv:1705.08903] [INSPIRE].

[43] S. Frixione, E. Laenen, P. Motylinski and B.R. Webber, Single-top production in MC@NLO, JHEP 03 (2006) 092 [hep-ph/0512250] [INSPIRE].

[44] S. Alioli, P. Nason, C. Oleari and E. Re, NLO single-top production matched with shower in POWHEG: s- and t-channel contributions, JHEP 09 (2009) 111 [Erratum ibid. 02 (2010) 011] [arXiv:0907.4076] [INSPIRE].

[45] E. Bothmann, F. Krauss and M. Schönherr, Single top-quark production with SHERPA, Eur. Phys. J. C 78 (2018) 220 [arXiv:1711.02568] [InSPIRE].

[46] J. Alwall et al., The automated computation of tree-level and next-to-leading order differential cross sections and their matching to parton shower simulations, JHEP $\mathbf{0 7}$ (2014) 079 [arXiv: 1405.0301] [InSPIRE].

[47] T. Carli et al., A posteriori inclusion of parton density functions in NLO QCD final-state calculations at hadron colliders: The APPLGRID Project, Eur. Phys. J. C 66 (2010) 503 [arXiv:0911.2985] [INSPIRE].

[48] V. Bertone, R. Frederix, S. Frixione, J. Rojo and M. Sutton, aMCfast: automation of fast NLO computations for PDF fits, JHEP 08 (2014) 166 [arXiv: 1406.7693] [INSPIRE].

[49] V. Bertone, S. Carrazza and N.P. Hartland, APFELgrid: a high performance tool for parton density determinations, Comput. Phys. Commun. 212 (2017) 205 [arXiv:1605.02070] [INSPIRE].

[50] T. Han, G. Valencia and S. Willenbrock, Structure function approach to vector boson scattering in pp collisions, Phys. Rev. Lett. 69 (1992) 3274 [hep-ph/9206246] [INSPIRE].

[51] NNPDF collaboration, Unbiased global determination of parton distributions and their uncertainties at NNLO and at LO, Nucl. Phys. B 855 (2012) 153 [arXiv:1107.2652] [INSPIRE].

[52] L.A. Harland-Lang, A.D. Martin, P. Motylinski and R.S. Thorne, Parton distributions in the LHC era: MMHT 2014 PDFs, Eur. Phys. J. C 75 (2015) 204 [arXiv:1412.3989] [INSPIRE].

[53] T.-J. Hou et al., Progress in the CTEQ-TEA NNLO global QCD analysis, arXiv:1908.11394 [INSPIRE].

[54] J. Butterworth et al., PDF 4 LHC recommendations for LHC Run II, J. Phys. G 43 (2016) 023001 [arXiv: 1510.03865] [INSPIRE].

[55] CDF and D0 collaborations, Tevatron Combination of Single-Top-Quark Cross Sections and Determination of the Magnitude of the Cabibbo-Kobayashi-Maskawa Matrix Element $V_{t b}$, Phys. Rev. Lett. 115 (2015) 152003 [arXiv:1503.05027] [INSPIRE].

[56] P. Tepel, Measurement of the Inclusive and Fiducial Cross-Section of Single Top-Quark t-Channel Events in pp Collisions at $\sqrt{s}=8 \mathrm{TeV}$, arXiv:1411.7627 [INSPIRE]. 
[57] R. Frederix, S. Frixione, V. Hirschi, D. Pagani, H.S. Shao and M. Zaro, The automation of next-to-leading order electroweak calculations, JHEP 07 (2018) 185 [arXiv:1804.10017] [INSPIRE].

[58] NNPDF collaboration, Illuminating the photon content of the proton within a global PDF analysis, SciPost Phys. 5 (2018) 008 [arXiv:1712.07053] [INSPIRE].

[59] R. Frederix, D. Pagani and I. Tsinikos, Precise predictions for single-top production: the impact of EW corrections and QCD shower on the t-channel signature, JHEP 09 (2019) 122 [arXiv: 1907.12586] [INSPIRE].

[60] NNPDF collaboration, Parton distributions for the LHC Run II, JHEP 04 (2015) 040 [arXiv: 1410.8849$]$ [INSPIRE].

[61] M. Cacciari, M. Greco and P. Nason, The $p_{T}$ spectrum in heavy flavor hadroproduction, JHEP 05 (1998) 007 [hep-ph/9803400] [inSPIRE].

[62] S. Forte, E. Laenen, P. Nason and J. Rojo, Heavy quarks in deep-inelastic scattering, Nucl. Phys. B 834 (2010) 116 [arXiv: 1001.2312] [InSPIRE].

[63] S. Forte, D. Napoletano and M. Ubiali, Higgs production in bottom-quark fusion in a matched scheme, Phys. Lett. B 751 (2015) 331 [arXiv:1508.01529] [INSPIRE].

[64] S. Forte, D. Napoletano and M. Ubiali, Higgs production in bottom-quark fusion: matching beyond leading order, Phys. Lett. B 763 (2016) 190 [arXiv:1607.00389] [INSPIRE].

[65] S. Forte, D. Napoletano and M. Ubiali, $Z$ boson production in bottom-quark fusion: a study of b-mass effects beyond leading order, Eur. Phys. J. C 78 (2018) 932 [arXiv:1803.10248] [INSPIRE].

[66] F. Maltoni, G. Ridolfi and M. Ubiali, b-initiated processes at the LHC: a reappraisal, JHEP 07 (2012) 022 [Erratum ibid. 04 (2013) 095] [arXiv: 1203.6393] [INSPIRE].

[67] C. Degrande, M. Ubiali, M. Wiesemann and M. Zaro, Heavy charged Higgs boson production at the LHC, JHEP 10 (2015) 145 [arXiv:1507.02549] [INSPIRE].

[68] R.D. Ball, M. Bonvini and L. Rottoli, Charm in Deep-Inelastic Scattering, JHEP 11 (2015) 122 [arXiv: 1510.02491] [INSPIRE].

[69] R.D. Ball et al., Intrinsic charm in a matched general-mass scheme, Phys. Lett. B 754 (2016) 49 [arXiv: 1510.00009] [INSPIRE].

[70] NNPDF collaboration, A determination of the Charm Content of the Proton, Eur. Phys. J. C 76 (2016) 647 [arXiv: 1605.06515] [INSPIRE].

[71] S. Forte, T. Giani and D. Napoletano, Fitting the b-quark PDF as a massive-b scheme: Higgs production in bottom fusion, Eur. Phys. J. C 79 (2019) 609 [arXiv:1905.02207] [INSPIRE].

[72] G. D'Agostini, On the use of the covariance matrix to fit correlated data, Nucl. Instrum. Meth. A 346 (1994) 306 [inSPIRE].

[73] NNPDF collaboration, Fitting Parton Distribution Data with Multiplicative Normalization Uncertainties, JHEP 05 (2010) 075 [arXiv:0912.2276] [INSPIRE].

[74] A. Guffanti and J. Rojo, Top production at the LHC: The impact of PDF uncertainties and correlations, Nuovo Cim. C 033 (2010) 65 [arXiv: 1008.4671] [INSPIRE].

[75] NNPDF collaboration, Precision determination of the strong coupling constant within a global PDF analysis, Eur. Phys. J. C 78 (2018) 408 [arXiv: 1802.03398] [INSPIRE]. 
[76] New Muon collaboration, Accurate measurement of $F_{2}^{d} / F_{2}^{p}$ and $R^{d}-R^{p}$, Nucl. Phys. B 487 (1997) 3 [hep-ex/9611022] [INSPIRE].

[77] New Muon collaboration, Measurement of the proton and deuteron structure functions, $F_{2}^{p}$ and $F_{2}^{d}$, and of the ratio $\sigma_{L} / \sigma_{T}$, Nucl. Phys. B 483 (1997) 3 [hep-ph/9610231] [INSPIRE].

[78] L.W. Whitlow, E.M. Riordan, S. Dasu, S. Rock and A. Bodek, Precise measurements of the proton and deuteron structure functions from a global analysis of the SLAC deep inelastic electron scattering cross-sections, Phys. Lett. B 282 (1992) 475 [INSPIRE].

[79] BCDMS collaboration, A High Statistics Measurement of the Proton Structure Functions $F_{2}\left(x, Q^{2}\right)$ and $R$ from Deep Inelastic Muon Scattering at High $Q^{2}$, Phys. Lett. B 223 (1989) 485 [INSPIRE].

[80] BCDMS collaboration, A High Statistics Measurement of the Deuteron Structure Functions $F_{2}\left(x, Q^{2}\right)$ and $R$ From Deep Inelastic Muon Scattering at High $Q^{2}$, Phys. Lett. B 237 (1990) 592 [INSPIRE].

[81] CHORUS collaboration, Measurement of nucleon structure functions in neutrino scattering, Phys. Lett. B 632 (2006) 65 [INSPIRE].

[82] NuTeV collaboration, Precise Measurement of Dimuon Production Cross-Sections in $\nu_{\mu} \mathrm{Fe}$ and $\bar{\nu}_{\mu}$ Fe Deep Inelastic Scattering at the Tevatron., Phys. Rev. D 64 (2001) 112006 [hep-ex/0102049] [INSPIRE].

[83] D.A. Mason, Measurement of the strange-antistrange asymmetry at NLO in QCD from NuTeV dimuon data, Ph.D. thesis, Oregon University, U.S.A. (2006), [DOI].

[84] H1 and ZEUS collaborations, Combination of measurements of inclusive deep inelastic $e^{ \pm} p$ scattering cross sections and QCD analysis of HERA data, Eur. Phys. J. C 75 (2015) 580 [arXiv: 1506.06042] [INSPIRE].

[85] H1 and ZEUS collaborations, Combination and QCD Analysis of Charm Production Cross Section Measurements in Deep-Inelastic ep Scattering at HERA, Eur. Phys. J. C 73 (2013) 2311 [arXiv: 1211.1182] [INSPIRE].

[86] H1 collaboration, Measurement of the Charm and Beauty Structure Functions using the H1 Vertex Detector at HERA, Eur. Phys. J. C 65 (2010) 89 [arXiv:0907.2643] [InSPIRE].

[87] ZEUS collaboration, Measurement of beauty and charm production in deep inelastic scattering at HERA and measurement of the beauty-quark mass, JHEP 09 (2014) 127 [arXiv:1405.6915] [INSPIRE].

[88] NuSea collaboration, Absolute Drell-Yan dimuon cross-sections in $800 \mathrm{GeV} / \mathrm{c}$ pp and pd collisions, hep-ex/0302019 [INSPIRE].

[89] J.C. Webb, Measurement of continuum dimuon production in 800-GeV/C proton nucleon collisions, Ph.D. thesis, New Mexico State University, U.S.A. (2003), hep-ex/0301031 [DOI] [INSPIRE].

[90] NuSEa collaboration, Improved measurement of the $\bar{d} / \bar{u}$ asymmetry in the nucleon sea, Phys. Rev. D 64 (2001) 052002 [hep-ex/0103030] [InSPIRE].

[91] G. Moreno et al., Dimuon production in proton-copper collisions at $\sqrt{s}=38.8 \mathrm{GeV}$, Phys. Rev. D 43 (1991) 2815 [INSPIRE].

[92] CDF collaboration, Measurement of $d \sigma / d y$ of Drell-Yan $e^{+} e^{-}$pairs in the $Z$ Mass Region from $p \bar{p}$ Collisions at $\sqrt{s}=1.96 \mathrm{TeV}$, Phys. Lett. B 692 (2010) 232 [arXiv:0908.3914] [INSPIRE]. 
[93] D0 collaboration, Measurement of the Shape of the Boson Rapidity Distribution for $p \bar{p} \rightarrow Z / \gamma^{*} \rightarrow e^{+} e^{-}+X$ Events Produced at $\sqrt{s}$ of 1.96-TeV, Phys. Rev. D 76 (2007) 012003 [hep-ex/0702025] [INSPIRE].

[94] D0 collaboration, Measurement of the Muon Charge Asymmetry in $p \bar{p} \rightarrow W+X \rightarrow \mu \nu+X$ Events at $\sqrt{s}=1.96 \mathrm{TeV}$, Phys. Rev. D 88 (2013) 091102 [arXiv:1309.2591] [INSPIRE].

[95] D0 collaboration, Measurement of the electron charge asymmetry in $p \bar{p} \rightarrow W+X \rightarrow e \nu+X$ decays in $p \bar{p}$ collisions at $\sqrt{s}=1.96$ TeV, Phys. Rev. D 91 (2015) 032007 [Erratum ibid. D 91 (2015) 079901] [arXiv:1412.2862] [INSPIRE].

[96] ATLAS collaboration, Measurement of the high-mass Drell-Yan differential cross-section in pp collisions at $\sqrt{s}=7 \mathrm{TeV}$ with the ATLAS detector, Phys. Lett. B 725 (2013) 223 [arXiv:1305.4192] [INSPIRE].

[97] ATLAS collaboration, Measurement of the low-mass Drell-Yan differential cross section at $\sqrt{s}=7 \mathrm{TeV}$ using the ATLAS detector, JHEP 06 (2014) 112 [arXiv:1404.1212] [inSPIRE].

[98] ATLAS collaboration, Measurement of the inclusive $W^{ \pm}$and $Z / \gamma^{*}$ cross sections in the $e$ and $\mu$ decay channels in pp collisions at $\sqrt{s}=7 \mathrm{TeV}$ with the ATLAS detector, Phys. Rev. D 85 (2012) 072004 [arXiv:1109.5141] [INSPIRE].

[99] ATLAS collaboration, Precision measurement and interpretation of inclusive $W^{+}, W^{-}$and $Z / \gamma^{*}$ production cross sections with the ATLAS detector, Eur. Phys. J. C 77 (2017) 367 [arXiv: 1612.03016] [INSPIRE].

[100] ATLAS collaboration, Measurement of the transverse momentum and $\phi_{\eta}^{*}$ distributions of Drell-Yan lepton pairs in proton-proton collisions at $\sqrt{s}=8 \mathrm{TeV}$ with the ATLAS detector, Eur. Phys. J. C 76 (2016) 291 [arXiv:1512.02192] [INSPIRE].

[101] ATLAS collaboration, Measurement of inclusive jet and dijet production in pp collisions at $\sqrt{s}=7 \mathrm{TeV}$ using the ATLAS detector, Phys. Rev. D 86 (2012) 014022 [arXiv:1112.6297] [INSPIRE].

[102] ATLAS collaboration, Measurement of the t$t \bar{t}$ production cross-section using e $\mu$ events with b-tagged jets in pp collisions at $\sqrt{s}=7$ and $8 \mathrm{TeV}$ with the ATLAS detector, Eur. Phys. J. C 74 (2014) 3109 [arXiv:1406.5375] [INSPIRE].

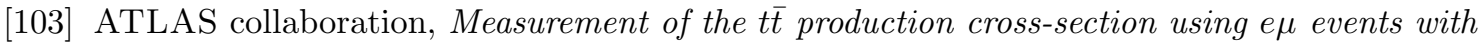
b-tagged jets in pp collisions at $\sqrt{s}=13 \mathrm{TeV}$ with the ATLAS detector, Phys. Lett. B 761 (2016) 136 [Erratum ibid. B 772 (2017) 879] [arXiv: 1606.02699] [INSPIRE].

[104] ATLAS collaboration, Measurements of top-quark pair differential cross-sections in the lepton+jets channel in pp collisions at $\sqrt{s}=8 \mathrm{TeV}$ using the ATLAS detector, Eur. Phys. J. C 76 (2016) 538 [arXiv:1511.04716] [INSPIRE].

[105] CMS collaboration, Measurement of the Electron Charge Asymmetry in Inclusive $W$ Production in pp Collisions at $\sqrt{s}=7$ TeV, Phys. Rev. Lett. 109 (2012) 111806 [arXiv: 1206.2598] [INSPIRE].

[106] CMS collaboration, Measurement of the Muon Charge Asymmetry in Inclusive $p p \rightarrow W+X$ Production at $\sqrt{s}=7 \mathrm{TeV}$ and an Improved Determination of Light Parton Distribution Functions, Phys. Rev. D 90 (2014) 032004 [arXiv: 1312.6283] [INSPIRE].

[107] CMS collaboration, Measurement of the Differential and Double-Differential Drell-Yan Cross Sections in Proton-Proton Collisions at $\sqrt{s}=7 \mathrm{TeV}$, JHEP 12 (2013) 030 [arXiv: 1310.7291] [INSPIRE]. 
[108] CMS collaboration, Measurement of the differential cross section and charge asymmetry for inclusive $p p \rightarrow W^{ \pm}+X$ production at $\sqrt{s}=8$ TeV, Eur. Phys. J. C 76 (2016) 469 [arXiv: 1603.01803] [INSPIRE].

[109] CMS collaboration, Measurement of the $Z$ boson differential cross section in transverse momentum and rapidity in proton-proton collisions at 8 TeV, Phys. Lett. B 749 (2015) 187 [arXiv: 1504.03511] [INSPIRE].

[110] CMS collaboration, Measurements of Differential Jet Cross Sections in Proton-Proton Collisions at $\sqrt{s}=7 \mathrm{TeV}$ with the CMS Detector, Phys. Rev. D 87 (2013) 112002 [Erratum ibid. D 87 (2013) 119902] [arXiv: 1212.6660] [INSPIRE].

[111] CMS collaboration, Measurement of the $t \bar{t}$ production cross section in the e $\mu$ channel in proton-proton collisions at $\sqrt{s}=7$ and $8 \mathrm{TeV}$, JHEP 08 (2016) 029 [arXiv:1603.02303] [INSPIRE].

[112] CMS collaboration, Measurement of the top quark pair production cross section in proton-proton collisions at $\sqrt{s}=13 \mathrm{TeV}$, Phys. Rev. Lett. 116 (2016) 052002 [arXiv: 1510.05302] [INSPIRE].

[113] CMS collaboration, Measurement of the differential cross section for top quark pair production in pp collisions at $\sqrt{s}=8 \mathrm{TeV}$, Eur. Phys. J. C 75 (2015) 542 [arXiv: 1505.04480] [INSPIRE].

[114] LHCb collaboration, Inclusive $W$ and $Z$ production in the forward region at $\sqrt{s}=7 \mathrm{TeV}$, JHEP 06 (2012) 058 [arXiv: 1204.1620] [INSPIRE].

[115] LHCb collaboration, Measurement of the cross-section for $Z \rightarrow e^{+} e^{-}$production in $p p$ collisions at $\sqrt{s}=7 \mathrm{TeV}$, JHEP 02 (2013) 106 [arXiv:1212.4620] [INSPIRE].

[116] LHCb collaboration, Measurement of the forward $Z$ boson production cross-section in pp collisions at $\sqrt{s}=7 \mathrm{TeV}$, JHEP 08 (2015) 039 [arXiv: 1505.07024] [INSPIRE].

[117] LHCb collaboration, Measurement of forward $W$ and $Z$ boson production in pp collisions at $\sqrt{s}=8 \mathrm{TeV}$, JHEP 01 (2016) 155 [arXiv:1511.08039] [INSPIRE].

[118] Particle Data Group collaboration, Review of Particle Physics, Phys. Rev. D 98 (2018) 030001 [INSPIRE].

[119] LHC Higgs Cross Section Working Group collaboration, Handbook of LHC Higgs Cross Sections: 4. Deciphering the Nature of the Higgs Sector, arXiv:1610.07922 [INSPIRE].

[120] V. Bertone, S. Carrazza and J. Rojo, APFEL: A PDF Evolution Library with QED corrections, Comput. Phys. Commun. 185 (2014) 1647 [arXiv:1310.1394] [INSPIRE].

[121] Z. Kassabov, Reportengine: A framework for declarative data analysis, (February 2019) [DOI].

[122] ATLAS collaboration, Measurement of $W^{ \pm}$and $Z$-boson production cross sections in pp collisions at $\sqrt{s}=13 \mathrm{TeV}$ with the ATLAS detector, Phys. Lett. B 759 (2016) 601 [arXiv: 1603.09222] [INSPIRE].

[123] M.L. Mangano et al., Physics at a 100 TeV pp Collider: Standard Model Processes, CERN Yellow Rep. (2017) 1 [arXiv:1607.01831] [InSPIRE]. 